\title{
A NON-CONFORMING GENERALIZED FINITE ELEMENT METHOD FOR TRANSMISSION PROBLEMS
}

By

\section{Anna L. Mazzucato, Victor Nistor, and Qingqin Qu}

\section{IMA Preprint Series \#2409}

(September 2012)

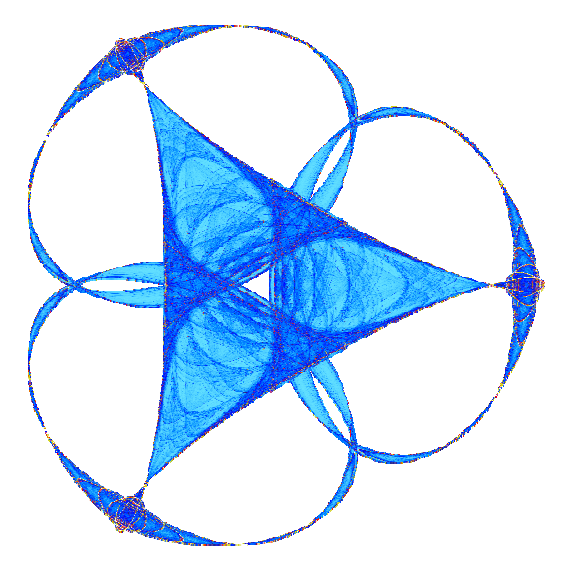

INSTITUTE FOR MATHEMATICS AND ITS APPLICATIONS

UNIVERSITY OF MINNESOTA 400 Lind Hall

207 Church Street S.E.

Minneapolis, Minnesota 55455-0436

Phone: 612-624-6066 Fax: 612-626-7370

URL: http://www.ima.umn.edu 


\section{A NON-CONFORMING GENERALIZED FINITE ELEMENT METHOD FOR TRANSMISSION PROBLEMS}

By

\section{Anna L. Mazzucato, Victor Nistor, and Qingqin Qu}

\section{IMA Preprint Series \#2409}

(September 2012)

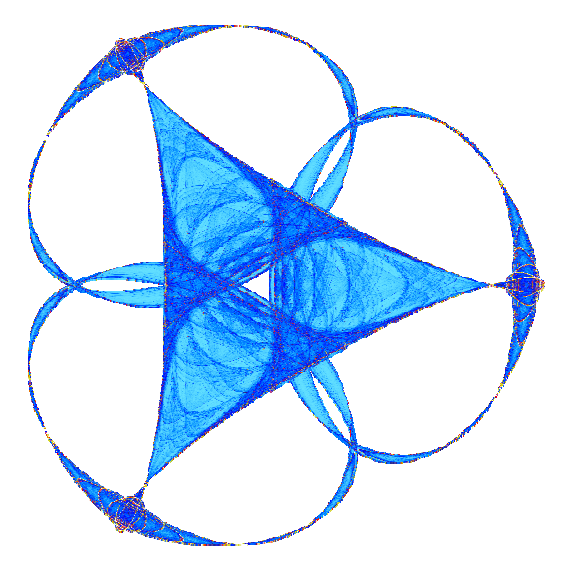

INSTITUTE FOR MATHEMATICS AND ITS APPLICATIONS

UNIVERSITY OF MINNESOTA 400 Lind Hall

207 Church Street S.E.

Minneapolis, Minnesota 55455-0436

Phone: 612-624-6066 Fax: 612-626-7370

URL: http://www.ima.umn.edu 


\title{
A NON-CONFORMING GENERALIZED FINITE ELEMENT METHOD FOR TRANSMISSION PROBLEMS
}

\author{
ANNA L. MAZZUCATO*, VICTOR NISTOR ${ }^{\dagger}$, AND QINGQIN QU
}

\begin{abstract}
We obtain "quasi-optimal rates of convergence" for transmission (interface) problems on domains with smooth, curved boundaries using a non-conforming Generalized Finite Element Method (GFEM). More precisely, we study the strongly elliptic problem $P u:=-\sum \partial_{j}\left(A^{i j} \partial_{i} u\right)=f$ in a smooth bounded domain $\Omega$ with Dirichlet boundary conditions. The coefficients $A^{i j}$ are piecewise smooth, possibly with jump discontinuities along a smooth, closed surface $\Gamma$, called the interface, which does not intersect the boundary of the domain. We consider a sequence of approximation spaces $S_{\mu}$ satisfying two conditions: (1) nearly zero boundary and interface matching, (2) approximability, which are similar to those in Babuška, Nistor, and Tarfulea, J. Comput. Appl. Math. (2008). Then, if $u_{\mu} \in S_{\mu}, \mu \geq 1$, is a sequence of Galerkin approximations of the solution $u$ to the interface problem, the approximation error $\left\|u-u_{\mu}\right\|_{\hat{H}^{1}(\Omega)}$ is of order $O\left(h_{\mu}^{m}\right)$, where $h_{\mu}^{m}$ is the typical size of the elements in $S^{\mu}$ and $\hat{H}^{1}$ is the Sobolev space of functions in $H^{1}$ on each side of the interface. We give an explicit construction of GFEM spaces $S_{\mu}$ for which our two assumptions are satisfied, and hence for which the quasi-optimal rates of convergence hold and present a numerical test.
\end{abstract}

Key words. strongly elliptic equations, interface problems, transmission conditions, partition of unity, generalized finite element, optimal rate of convergence

AMS subject classifications. 35J25,65N30,65N15

Introduction. Let us consider the second order elliptic equation

$$
P u:=-\operatorname{div}(A \nabla u):=-\sum_{i, j=1}^{n} \partial_{j}\left(A^{i j} \partial_{i} u\right)=f \text { in } \Omega,\left.\quad u\right|_{\partial \Omega}=0,
$$

where $\Omega \subset \mathbb{R}^{d}$ is a bounded domain with a smooth boundary $\partial \Omega$ and $P$ is a secondorder, scalar differential operator with real coefficients. We assume that the coefficient matrix $A=\left(A^{i j}\right)_{n \times n}$ is piecewise smooth with only jump discontinuities across a finite number of smooth, closed surfaces, the union of which is called the interface, and is denoted by $\Gamma$. In this work, we assume that the interface does not intersect the boundary of $\Omega, \partial \Omega \cap \Gamma=\emptyset$. The interface partitions $\Omega$ into a finite number of smooth subdomains $\Omega_{j}$. We say that $u$ is locally strong solution of $(0.1)$ if $u \in \mathcal{C}^{2}\left(\Omega_{j}\right) \cap \mathcal{C}\left(\bar{\Omega}_{j}\right)$ solves (0.1) pointwise away from the interface. When the coefficients are piecewise smooth, any locally strong solution is a weak solution if it satisfies certain jump or transmission conditions at the interface $\Gamma$. (See Equation (0.8) below.)

The problem (0.1) is then called a transmission (or interface) problem, and it appears in many practical applications. In particular, transmission problems arise when the underlying physical problem is formulated for a body that consists of more than one material. Because of this connection, extensive research has been done on the transmission problem. For instance, the classical approach using isoparametric elements was extended to interfaces by Barrett and Elliott in [10]. This work extends the earlier work on the Dirichlet problem [9] by the same authors and underscores the similarities between the treatment of boundary and interface problems

\footnotetext{
*Mathematics Department, Penn State University, University Park, PA 16802, USA, (alm24@psu.edu)

$\dagger$ Mathematics Department, Penn State University, University Park, PA 16802, USA, (nistor@math.psu.edu)

¥ Mathematics Department, Penn State University, University Park, PA 16802, USA, (qu@math.psu.edu). Corresponding author.
} 


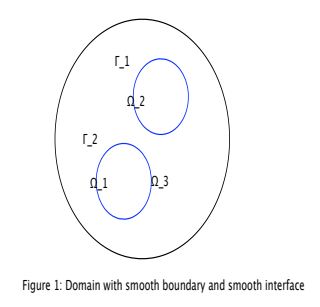

FIG. 0.1. Domain with smooth boundary and smooth interface

using isoparametric elements. This similarity is also apparent in the present work for GFEM. Optimal a priori estimates for higher-order FEMs were obtained by $\mathrm{Li}$, Melenk, Wohlmuth, and Zhu in [27]. In that paper, the authors consider the brocken Sobolev spaces $\hat{H}(\Omega)$ in the treatment of their error estimates. The brocken Sobolev spaces and their weighted analogues also play an important role in the regularity estimates in polygonal and polyhedral domains for interface problems obtained in $[26,40]$, where also applications to $h^{m}$-quasi-optimal rates of convergence for FEM were obtained. Multigrid methods for solving interface problems were obtained in [13, 41, 42, 43]. A systematic treatment of transmission problems is provided in [29]. See also $[16,24,23,28,31,22]$ and the references cited therein.

Let us now explain our framework in more detail. We assume that the coefficients $A^{i j}$ are bounded and that the resulting operator is uniformly strongly elliptic, that is:

$$
\alpha\|\xi\|^{2} \leq \sum_{i, j=1}^{n} A^{i j}(x) \xi_{i} \xi_{j} \leq C\|\xi\|^{2}
$$

for some constants $\alpha>0$ and $C>0$ independent of $x \in \bar{\Omega}$ and $\xi \in \mathbb{R}^{n}$. We also assume that we are given a decomposition

$$
\bar{\Omega}=\bigcup_{j=1}^{K} \bar{\Omega}_{j} \subset \mathbb{R}^{n},
$$

where $\Omega_{j}$ are disjoint subdomains with smooth boundaries that define the interface $\Gamma=\left(\bigcup_{j=1}^{K} \partial \Omega_{j}\right) \backslash \partial \Omega$. We further assume $\Gamma$ to be the union of a finite number of closed, smooth simple surfaces $\Gamma_{j}$ that do not intersect each other nor the boundary $\partial \Omega$. See Figure 0.1 for a typical example, in which $K=3$ and $\Gamma$ is the disjoint union of its two disjoint components $\Gamma_{1} \equiv \partial \Omega_{1}$ and $\Gamma_{2} \equiv \partial \Omega_{2}$.

Our paper provides a treatment of the transmission problem (0.1) that yields the theoretical $h^{m}$-quasi-optimal rate of convergence for an associated discretization. In general, the solutions of transmission problems have lower regularity in the usual Sobolev spaces. We shall therefore introduce the broken Sobolev spaces $\hat{H}^{p}(\Omega)$ that are better adapted to transmission problems. They are defined by:

$$
\hat{H}^{p}(\Omega):=\left\{u \in L^{2}(\Omega), u \in H^{p}\left(\Omega_{j}\right), \text { for } j=1, \ldots, K\right\},
$$

with norm and seminorm respectively

$$
\|u\|_{\hat{H}^{p}(\Omega)}^{2}=\sum_{j=1}^{K}\|u\|_{H^{p}\left(\Omega_{j}\right)}^{2}, \quad \text { and } \quad|u|_{\hat{H}^{p}(\Omega)}^{2}=\sum_{j=1}^{K}|u|_{H^{p}\left(\Omega_{j}\right)}^{2} .
$$


Also, we shall denote by

$$
\||u|\|^{2}:=\|u\|_{L^{2}(\partial \Omega)}^{2}+\left\|u_{+}-u_{-}\right\|_{L^{2}(\Gamma)}^{2}
$$

where $u_{+}, u_{-}$are the traces of $u \in \hat{H}^{1}(\Omega)$ on each side of the interface. The trace operator is well-defined on $\hat{H}^{s}(\Omega), s>1 / 2$, as it is well defined on $H^{s}\left(\Omega_{j}\right)$ for each $j=1, \ldots, K$. We observe that, by density of $C^{\infty}\left(\bar{\Omega}_{j}\right)$ in $H^{s}\left(\Omega_{j}\right)$, the trace agrees with the non-tangential limit of $u$ at $\partial \Omega_{j}$, i.e., on one side of the interface. (For a definition of non-tangential limit, we refer e.g. to [36, page 197].)

We shall consider a sequence $S_{\mu} \subset \hat{H}^{1}(\Omega)$ of generalized finite element (GFE) spaces satisfying

$$
\operatorname{dim}\left(S_{\mu}\right) \sim h_{\mu}^{-n}
$$

where $n$ is the dimension of the space, and $h_{\mu} \rightarrow 0$ is the typical size of the elements in $S_{\mu}$, as in [7], for example. We will denote by $u_{\mu}$ the projection of the solution $u$ to (0.1) onto $S_{\mu}$ in the $\hat{H}^{1}$-seminorm (see Equation (1.4)). We shall further assume that the spaces $S_{\mu}$ are given in terms of a fixed integer $m$, which plays the role of the approximation order in this work and satisfy Assumptions 1.3 (nearly zero boundary conditions and nearly interface matching) and 1.4 (approximability). Under these conditions, we obtain the theoretical $h^{m}$-quasi-optimal rate of convergence, which is the main result of this paper.

THEOREM 1. Let $S_{\mu} \subset \hat{H}^{1}(\Omega)$ be a sequence of finite-dimensional spaces, satisfying the nearly zero boundary conditions and interface matching, and the approximability condition, for a sequence $h_{\mu} \rightarrow 0$. If $f \in \hat{H}^{m-1}(\Omega)$ and $m \geq 1$, then there exists a constant $C>0$, independent of $f$ and $\mu$, such that

$$
\left\|u-u_{\mu}\right\|_{\hat{H}^{1}(\Omega)} \leq C h_{\mu}^{m}\|f\|_{\hat{H}^{m-1}(\Omega)},
$$

where $u$ is the exact solution to (0.1), and $u_{\mu}$ is its approximation in the space $S_{\mu}$.

It is known that equation (0.1) has a unique (weak) solution in $H_{0}^{1}(\Omega):=H^{1}(\Omega) \cap$ $\left\{\left.u\right|_{\partial \Omega}=0\right\}$. As already recalled, the discontinuities of the coefficient matrix $A$ lead to the so called "transmission" or "jump conditions" at the interface $\Gamma$ :

$$
u_{+}=u_{-}, \quad D_{\nu+}^{P} u=D_{\nu-}^{P} u
$$

which are necessary conditions for any locally strong solution away of the interface to be a weak solution. Above, we label the traces (or non-tangential limits) $u_{+}, u_{-}$of $u$ at each side of the interface, and denote the respective conormal derivatives by $D_{\nu+}^{P}$ and $D_{\nu-}^{P}$, given by:

$$
D_{\nu \pm}^{P} u:=\sum_{i, j} \nu_{i} A^{i, j} \partial_{j} u_{ \pm}=\nu \cdot A \cdot \nabla u_{ \pm}
$$

where $\nu$ is the outer unit normal vector to the interface $\Gamma$. By outer normal here, we mean the outer normal to each subdomain $\Omega_{j}$. The labeling \pm is only for notational convenience and plays no role.

In this article, we study the problem (0.1) using the Generalized Finite Element method (GFEM), extending the work in [6] and [7] to transmission problems. The GFEM is a generalization of the meshless methods based on partitions of unity. The support of the partition of unity need not depend on any mesh or on the geometry of the domain $\Omega$. As an extension of FEM, the GFEM allows great flexibility 
in constructing trial spaces. In particular, the GFEM allows one to include a priori knowledge about the local behavior of the solution, and gives the option of constructing trial spaces of any desired regularity. For more information on the GFEM and associated partition of unity, see $[1,3,4,5,8,37]$ and references cited therein. Recent work showcases some of the advantages of the GFEM, as it applies to various fields $[18,19,34,38]$.

We next briefly review the main ideas of the GFEM. Given overlapping patches $\left\{\omega_{j}\right\}$ such that $\bar{\Omega}=\cup_{j=1}^{N} \bar{\omega}_{j}$, let $\left\{\psi_{j}\right\}$ be a partition of unity subordinate to these patches. On each patch, let function spaces $\Psi_{j}$ reflect the local approximability. Then the global GFE space $S$ is given by

$$
S=\left\{\sum_{j} \psi_{j} v_{j}, v_{j} \in \Psi_{j}\right\}
$$

Our local approximation spaces are polynomials or piecewise polynomials, depending on whether the patch intersects the boundary or the interface, which makes their implementation easier to carry out than for non-polynomial spaces.

Similarly to the case of the usual FEM, one of the major problems in the implementation of meshless methods is the enforcement of Dirichlet boundary conditions. As in the paper [7], we address the problem of enforcing Dirichlet boundary conditions in the GFEM framework. The classical FEM assumes that the trial subspace functions fulfill the boundary conditions. Nevertheless, in practice the construction implies difficulties when the boundary is curved. Nitsche's approach is to use subspace with nearly zero boundary conditions. This idea was first outlined by Nitsche [30], and further studied by Berger, Scott and Strang [11] and Scott [33], who called it "interpolated boundary conditions". In this paper, we also use nearly zero boundary conditions. As discussed in Section 4, this method of enforcing boundary conditions is straightforward to realize in practice. In fact, our code for the GFEM is very simple (available on the authors' webpage).

For transmission problems an additional difficulty arises from the interface. Because of the loss of regularity in the problem (0.1), in order to get a good local approximation space, one needs to include transmission conditions in the construction of the space. Again, it is difficult to impose the transmission conditions exactly on the approximate solution, when the interface is curved. We employ polynomials to approximate the interface, and then construct the local approximation spaces of piecewise polynomials that are nearly continuous across the approximate interface and allow jumps in their derivatives. While superficially, the difficulties encountered in enforcing the Dirichlet boundary conditions are mirrored in similar difficulties in enforcing the transmission conditions at the interface, in fact, both theoretically and from an implementation point of view, the treatment of interfaces requires a new approach to deal with the discontinuities of the trial functions across the interface. The approach we used has certain points in common with the Isoparametric Finite Element Method (IFEM) since both of them use polynomials to approximate the boundary. But our construction is also different from the IFEM. See [15, 17], or [35] for the recent work and applications on IFEM. In the IFEM the domain is approximated by $\Omega_{h}$ and the approximate solution $u_{h}$ is sought in a subspace $V_{h} \subset H_{0}^{1}\left(\Omega_{h}\right)$, so the boundary condition $u=0$ on $\partial \Omega$ is approximated by $u_{h}=0$ on $\partial \Omega_{h}$. Differently than in the IFEM however, we do not need to map to a reference domain, which may entail a non-polynomial change of coordinates.

Although we restrict our attention in this paper to scalar equations with Dirichlet boundary conditions, our method can be generalized to other type of boundary 
conditions, such as Neumann boundary conditions, and to strongly elliptic systems. Non-homogeneous boundary conditions can be treated exactly like in [7], since the interface does not touch the boundary.

The paper is organized as follows. In the first section, we show that Assumptions 1.3 and 1.4 lead to $h^{m}$-quasi-optimal rates of convergence. In Section 2 we introduce the GFE spaces used in this paper, in particular, we introduce the conditions $\mathrm{A}\left(h_{\mu}\right)$, $\mathrm{B}, \mathrm{C}$, and D satisfied by the data that define our GFE spaces. In Section 3, we prove that conditions $\mathrm{A}\left(h_{\mu}\right), \mathrm{B}, \mathrm{C}$, and D imply Assumptions 1.3 and 1.4. In Section 4, numerical tests are provided to confirm our theoretical result.

1. Approximate Dirichlet boundary conditions. In this section we prove our main approximation result (Theorem 1.11) under the two Assumptions formulated below.

We recall that $\bar{\Omega}=\cup_{j=1}^{K} \bar{\Omega}_{j}$ is a bounded domain that is decomposed as the union of the closures of the disjoint domains $\Omega_{j}$. We also recall that the set $\Gamma:=$ $\cup_{j=1}^{K} \partial \Omega_{j} \backslash \partial \Omega$ is called the interface. We shall fix in what follows $m \in \mathbb{N}=\{1,2, \ldots\}$, which will play the role of the order of approximation.

In this paper, we shall assume that both the boundary $\partial \Omega$ and $\Gamma$ are smooth and do not intersect each other: $\partial \Omega \cap \Gamma=\emptyset$.

As already mentioned, any locally strong solution to (0.1) (i.e., any solution in $\left.\mathcal{C}^{2}\left(\Omega_{j}\right) \cap \mathcal{C}\left(\bar{\Omega}_{j}\right)\right)$ satisfies the Dirichlet problem for equation (0.1) in weak form only if it satisfies the following interface problem

$$
\left\{\begin{aligned}
P u & =f & & \text { in } \Omega \\
u & =0 & & \text { on } \partial \Omega \\
u_{+} & =u_{-} & & \text {on } \Gamma \\
D_{\nu+}^{P} u & =D_{\nu-}^{P} u & & \text { on } \Gamma,
\end{aligned}\right.
$$

where $u_{+}, u_{-}$are the traces of $u$ on each side of the interface and $D_{\nu+}^{P} u, D_{\nu-}^{P} u$ their conormal derivatives (given in (0.9)). The transmission conditions are hence a consequence of the weak formulation, and will always be considered as part of Equation (1.1).

We want to approximate $u$ with functions $u_{\mu} \in S_{\mu}, \mu \in \mathbb{N}$, where $S_{\mu} \subset \hat{H}^{1}(\Omega)$ is a sequence of finite dimensional subspaces that satisfy Assumptions 1.3 and 1.4 below.

First, let us recall the broken Sobolev spaces given in (0.4) and (0.5). Let

$$
B(u, v)=\sum_{i, j}^{n} \sum_{k}^{K} \int_{\Omega_{k}} A^{i j} \partial_{i} u \partial_{j} v d x
$$

be the bilinear form associated with problem (0.1). From the elliptic property (0.2), we have the following coercivity estimate

$$
\alpha|v|_{\hat{H}^{1}(\Omega)}^{2} \leq B(v, v), \text { for } v \in \hat{H}^{1}(\Omega) .
$$

We also have the following continuity property

$$
B(u, v) \leq|u|_{\hat{H}^{1}(\Omega)}|v|_{\hat{H}^{1}(\Omega)} .
$$

Assume now that we are given a sequence of finite-dimensional trial spaces $S_{\mu} \subset$ $\hat{H}^{1}(\Omega)$, and a numeric sequence $h_{\mu}>0, \mu \in \mathbb{N}, h_{\mu} \rightarrow 0$ as $\mu \rightarrow \infty$. 
REMARK 1.1. In the explicit construction of the spaces $S_{\mu}$ given later in the paper, $h_{\mu}$ will be the maximum size of the elements in the approximation space. We do not impose directly any condition on the minimum size of the patches, so no quasiuniform condition is required. However, the conditions on the partition of unity may imply a minimum condition on the diameters of the patches.

Then we define the discrete solution $u_{\mu}$ for $\mu$ large, as the solution of

$$
B\left(u_{\mu}, v_{\mu}\right)=\int_{\Omega} f(x) v_{\mu}(x) d x, \text { for all } v_{\mu} \in S_{\mu} .
$$

This is essentially the standard way to define the approximate solution for an elliptic problem. But note that, since the elements in $S_{\mu}$ do not exactly satisfy the Dirichlet boundary conditions on $\partial \Omega$ or the transmission conditions on $\Gamma$, the approximate solution $u_{\mu}$ is not a variational solution of the Dirichlet problem for (0.1) in the space $S_{\mu}$, contrary to what happens for conforming methods.

REMARK 1.2. In principle, one needs to know the geometry of $\Omega$ exactly in order to define the bilinear form B. In practice, even when the geometry is not known, it is usually given with some degree of accuracy. The relation between the influence of the approximation of the geometry, in particular the quadrature method used to numerically compute $B$, on the accuracy of the method is an interesting and important point. Results in this direction were obtained in [27, Corollary 4.9], where optimal error estimates were obtained taking into account also the error made by the approximation of the bilinear $B$ due to the limited knowledge of the interface. In that paper, also more freedom was allowed in approximating the interface. See also [10] for a related analysis, where isoparametric elements were used. These works deal with the Finite Element Method. Clearly this problem deserves further study in the case of the Generalized Finite Element Method. Also, it may be possible to use "boundary fitting curves" (transfinite blending functions) to match the boundary exactly (we owe this remark to J. Melenk). That approach would require a different treatment of the approximation issues than the one in this paper. We hope to address these points in future work.

Recall that we have denoted by $\||w|\|^{2}=\|w\|_{L^{2}(\partial \Omega)}^{2}+\left\|w_{+}-w_{-}\right\|_{L^{2}(\Gamma)}^{2}$.

Given a function $w \in \hat{H}^{1}(\Omega)$, we also define $\llbracket w \rrbracket: \partial \Omega \cup \Gamma \rightarrow \mathbb{C}$ as the function that is equal to $w$ on the boundary and to the jump $w_{+}-w_{-}$on the interface $\Gamma$ in trace sense. Thus $\||w|\|=\|\llbracket w \rrbracket\|_{L^{2}(\partial \Omega \cup \Gamma) \text {. }}$

We are now ready to state the conditions that the trial spaces $S_{\mu}$ must satisfy in order for the quasi-optimal rates of convergence for the approximate solution sequence to hold. We recall that $m$ is a fixed integer, which plays the role of the approximation order.

Assumption 1.3. Nearly zero boundary and nearly interface matching condition: For each fixed $m \geq 1$, there exists $C>0$, independent of $\mu$ and $v_{\mu}$, such that

$$
\left\|\left|v_{\mu}\right|\right\| \leq C h_{\mu}^{m}\left\|v_{\mu}\right\|_{\hat{H}^{1}(\Omega)}, \quad \forall \mu
$$

Assumption 1.4. Approximability: For each fixed $m \geq 1$, there exists $C>0$ such that for any $0 \leq r \leq m$, any $u \in \hat{H}^{r+1}(\Omega) \cap H_{0}^{1}(\Omega)$, and any $\mu$, there exists $u_{I} \in S_{\mu}$ satisfying

$$
\left|u-u_{I}\right|_{\hat{H}^{1}(\Omega)} \leq C h_{\mu}^{r}\|u\|_{\hat{H}^{r+1}(\Omega)} .
$$


In Section 2, we will give an example of a sequence of GFE spaces $S_{\mu}$ that satisfies these two assumptions (see Condition $\mathbf{A}\left(h_{\mu}\right)$, Condition $\mathbf{B}$, Condition $\mathbf{C}$ and Condition D).

REMARK 1.5. In this paper, we shall use the convention that $C$ indicates a generic positive constant, independent of $\mu$, which may be different each time when it is used and may depend on the approximation integer $m$.

We follow $[7,30,33]$ in proving our main result from these two assumptions. The proof is a consequence of several lemmas.

We start by establishing a needed norm equivalence in the finite dimensional trial spaces $S_{\mu}$. The following variant of the classical Poincaré's inequality that takes into account the jumps at the interface $\Gamma$ can be proved as in the classical case [15]. More details can be found $[10,12]$, where some very similar results have been proved. See also [14].

Lemma 1.6. There exists a constant $C$ depending only on $\Omega$ such that

$$
\|u\|_{\hat{H}^{1}(\Omega)}^{2} \leq C\left[|u|_{\hat{H}^{1}(\Omega)}^{2}+\||u|\|^{2}\right] .
$$

for all $u \in \hat{H}^{1}(\Omega)$.

We are ready not to prove the following equivalence of norms.

Lemma 1.7. Given a sequence of trial spaces $S_{\mu}$ satisfying Assumptions 1.3 and 1.4 , there exists $C>0$ independent of $\mu$, such that for any $\mu$ large enough and all $v_{\mu} \in S_{\mu}$

$$
\left\|v_{\mu}\right\|_{\hat{H}^{1}(\Omega)} \leq C\left|v_{\mu}\right|_{\hat{H}^{1}(\Omega)} .
$$

In particular, $\left|v_{\mu}\right|_{\hat{H}^{1}(\Omega)}$ and $\left\|v_{\mu}\right\|_{\hat{H}^{1}(\Omega)}$ are equivalent norms on $S_{\mu}$.

Proof. By definition, $\left|v_{\mu}\right|_{\hat{H}^{1}(\Omega)} \leq\left\|v_{\mu}\right\|_{\hat{H}^{1}(\Omega)}$. To establish the opposite inequality, we observe that from Lemma 1.6,

$$
\begin{aligned}
\left\|v_{\mu}\right\|_{\hat{H}^{1}(\Omega)}^{2} & \leq C\left[\left|v_{\mu}\right|_{\hat{H}^{1}(\Omega)}^{2}+\left\|\left|v_{\mu}\right|\right\|^{2}\right] \\
& \leq C\left|v_{\mu}\right|_{\hat{H}^{1}(\Omega)}^{2}+C h_{\mu}^{2 m}\left\|v_{\mu}\right\|_{\hat{H}^{1}(\Omega)}^{2},
\end{aligned}
$$

where the last inequality follows from Assumption 1.3. Therefore,

$$
\left\|v_{\mu}\right\|_{\hat{H}^{1}(\Omega)} \leq C\left(1-C h_{\mu}^{2 m}\right)^{-1 / 2}\left|v_{\mu}\right|_{\hat{H}^{1}(\Omega)} .
$$

Since $h_{\mu} \rightarrow 0$ as $\mu \rightarrow \infty$ by hypothesis, for $\mu$ large enough $\left(1-C h_{\mu}^{2 m}\right) \geq 1 / 2$, which gives the desired estimate.

We now turn to study the problem (1.1) in the broken Sobolev spaces. Recall that $D_{\nu}^{P}$ is the conormal derivative in the sense of trace at the boundary $\partial \Omega$, while $D_{\nu+}^{P}$ and $D_{\nu-}^{P}$ are the conormal derivatives on each side of the interface $\Gamma$, defined in equation (0.9).

Let $w_{\mu} \in S_{\mu}$, for $\mu$ large, be the solution of the following variational problem: for all $v_{\mu} \in S_{\mu}$,

$$
B\left(w_{\mu}, v_{\mu}\right)=\int_{\partial \Omega \cup \Gamma} D_{\nu}^{P} u \llbracket v_{\mu} \rrbracket d S(x),
$$

where $u$ is the solution of (1.1) and $D_{\nu}^{P} u$ on $\Gamma$ is given by $D_{\nu+}^{P} u=D_{\nu-}^{P} u$ in view of the transmission conditions that $u$ must satisfy. 
By Lemma 1.7, the Lax-Milgram Lemma (see e.g. [17]), and inequality (1.3), there exists a unique solution to problem (1.4) and (1.6).

LEMMA 1.8. Let $u$ be the solution of the transmission problem (1.1) and let $u_{\mu}$, and $w_{\mu}$ be as in Equation (1.4) and (1.6). Then $B\left(u-u_{\mu}-w_{\mu}, v_{\mu}\right)=0$ for all $v_{\mu} \in S_{\mu}$, and hence

$$
\left|u-u_{\mu}-w_{\mu}\right|_{\hat{H}^{1}(\Omega)} \leq C\left|u-v_{\mu}\right|_{\hat{H}^{1}(\Omega)} \text { for all } v_{\mu} \in S_{\mu} .
$$

Proof. Integrating by parts and using the transmission conditions for $u$ on the interface $\Gamma$, we have for all $v_{\mu} \in S_{\mu}$,

$$
\begin{aligned}
B\left(u, v_{\mu}\right): & =\sum_{i, j} \sum_{k} \int_{\Omega_{k}} A^{i j} \partial_{i} u \partial_{j} v_{\mu} d x \\
& =-\sum_{i, j} \sum_{k} \int_{\Omega_{k}} \partial_{j}\left(A^{i j} \partial_{i} u\right) v_{\mu} d x+\int_{\partial \Omega \cup \Gamma} D_{\nu}^{P} u \llbracket v_{\mu} \rrbracket d S(x) \\
& =\int_{\Omega} f v_{\mu} d x+\int_{\partial \Omega \cup \Gamma} D_{\nu}^{P} u \llbracket v_{\mu} \rrbracket d S(x)=B\left(u_{\mu}+w_{\mu}, v_{\mu}\right) .
\end{aligned}
$$

Using the continuity and coercivity of the bilinear form $B$, we obtain that, for all $v_{\mu} \in S_{\mu}$

$$
\begin{array}{r}
\alpha\left|u-u_{\mu}-w_{\mu}\right|_{\hat{H}^{1}(\Omega)}^{2} \leq B\left(u-u_{\mu}-w_{\mu}, u-u_{\mu}-w_{\mu}\right)=B\left(u-u_{\mu}-w_{\mu}, u-v_{\mu}\right) \\
\leq C\left|u-u_{\mu}-w_{\mu}\right|_{\hat{H}^{1}(\Omega)}\left|u-v_{\mu}\right|_{\hat{H}^{1}(\Omega)},
\end{array}
$$

which gives the desired estimate.

We assume now that the solution $u$ of $(1.1)$ is in $\hat{H}^{2}(\Omega)$. We will show later that this is the case for the problem at hand.

LEMma 1.9. For $\mu$ sufficiently large, the solution $w_{\mu}$ of problem (1.6) satisfies

$$
\left\|w_{\mu}\right\|_{\hat{H}^{1}(\Omega)} \leq C h_{\mu}^{m}\|u\|_{\hat{H}^{2}(\Omega)},
$$

where $C$ is a constant independent of $\mu$ and $u$.

Proof. From Lemma 1.7 and inequality (1.3) we have:

$$
\begin{aligned}
\left\|w_{\mu}\right\|_{\hat{H}^{1}(\Omega)}^{2} & \leq C\left|w_{\mu}\right|_{\hat{H}^{1}(\Omega)}^{2} \leq C \alpha^{-1} B\left(w_{\mu}, w_{\mu}\right) \\
& =C \alpha^{-1} \int_{\partial \Omega \cup \Gamma} D_{\nu}^{P} u \llbracket w_{\mu} \rrbracket d S(x) \\
& \leq C\left\|D_{\nu}^{P} u\right\|_{L^{2}(\partial \Omega \cup \Gamma)}\left\|\llbracket w_{\mu} \rrbracket\right\|_{L^{2}(\partial \Omega \cup \Gamma)} \\
& \leq C \sum_{j}\left\|D_{\nu}^{P} u\right\|_{L^{2}\left(\partial \Omega_{j}\right)}\left\|\left|w_{\mu}\right|\right\| \\
& \leq C h_{\mu}^{m} \sum_{j}\|u\|_{\hat{H}^{2}\left(\Omega_{j}\right)}\left\|w_{\mu}\right\|_{\hat{H}^{1}(\Omega)} \\
& \leq C h_{\mu}^{m}\|u\|_{\hat{H}^{2}(\Omega)}\left\|w_{\mu}\right\|_{\hat{H}^{1}(\Omega)},
\end{aligned}
$$

where we have used Assumption 1.3, the coercivity of $B$ (Equation (1.3)), and the trace estimate $\left\|D_{\nu}^{p} u\right\|_{L^{2}\left(\partial \Omega_{j}\right)} \leq C\|u\|_{H^{2}\left(\Omega_{j}\right)}$. 
Lemma 1.10. For $\mu$ sufficiently large, the solution $u_{\mu}$ of problem (1.4) satisfies

$$
\left\|u_{\mu}\right\|_{\hat{H}^{1}(\Omega)} \leq C\|u\|_{\hat{H}^{2}(\Omega)}
$$

where $C$ is a constant independent of $\mu$ and $u$.

Proof. Previous lemmas give

$$
\begin{aligned}
\left\|u_{\mu}\right\|_{\hat{H}^{1}(\Omega)}^{2} & \leq C\left|u_{\mu}\right|_{\hat{H}^{1}(\Omega)}^{2} \leq C \alpha^{-1} B\left(u_{\mu}, u_{\mu}\right) \\
& =C \alpha^{-1}\left[B\left(u, u_{\mu}\right)-B\left(w_{\mu}, u_{\mu}\right)\right] \\
& \leq C\|u\|_{\hat{H}^{1}(\Omega)}\left\|u_{\mu}\right\|_{\hat{H}^{1}(\Omega)}+C\left\|D_{\nu} u(x)\right\|_{L^{2}(\partial \Omega \cup \Gamma)}\left\|\left|u_{\mu}\right|\right\| \\
& \leq C\|u\|_{\hat{H}^{2}(\Omega)}\left\|u_{\mu}\right\|_{\hat{H}^{1}(\Omega)}+C h_{\mu}^{m}\|u\|_{\hat{H}^{2}(\Omega)}\left\|u_{\mu}\right\|_{\hat{H}^{1}(\Omega)} \\
& \leq C\|u\|_{\hat{H}^{2}(\Omega)}\left\|u_{\mu}\right\|_{\hat{H}^{1}(\Omega)} \leq C\|u\|_{\hat{H}^{2}(\Omega)}^{2} .
\end{aligned}
$$

In the second inequality, we used Assumption 1.3 and, in each subdomain, the trace estimate $\left\|D_{\nu}^{p} u(x)\right\|_{L^{2}\left(\partial \Omega_{j}\right)} \leq C\|u\|_{H^{2}\left(\Omega_{j}\right)}$. $\mathrm{C}$

We are now ready to prove our main result.

TheOREM 1.11. Given $m \geq 1$, let $S_{\mu} \subset \hat{H}^{1}(\Omega)$ be a sequence of finite dimensional subspaces satisfying Assumptions 1.3 and 1.4 for a sequence $h_{\mu} \rightarrow 0$. Let $f \in \hat{H}^{p-1}(\Omega), 1 \leq p \leq m$. Then the unique solutions $u$ and $u_{\mu}$ of problems (1.1) and (1.4), respectively, satisfy

$$
\left\|u-u_{\mu}\right\|_{\hat{H}^{1}(\Omega)} \leq C h_{\mu}^{p}\|u\|_{\hat{H}^{p+1}(\Omega)} \leq C_{1} h_{\mu}^{p}\|f\|_{\hat{H}^{p-1}(\Omega)},
$$

for a constant $C$ independent of $\mu$ and $f$.

Proof. Since $f \in \hat{H}^{p-1}(\Omega)$ and each $\Omega_{j}$ is a smooth domain with smooth boundary, by regularity results in [32], $u \in \hat{H}^{p+1}(\Omega)$, and $\|u\|_{\hat{H}^{p+1}(\Omega)} \leq C\|f\|_{\hat{H}^{p-1}(\Omega)}$. We assume $p=m$, for simplicity. Lemma 1.8, Lemma 1.9, and Assumption 1.4 give

$$
\begin{aligned}
\left|u-u_{\mu}\right|_{\hat{H}^{1}(\Omega)} & \leq\left|u-u_{\mu}-w_{\mu}\right|_{\hat{H}^{1}(\Omega)}+\left|w_{\mu}\right|_{\hat{H}^{1}(\Omega)} \mid \\
& \leq\left|u-u_{I}\right|_{\hat{H}^{1}(\Omega)}+C h_{\mu}^{m}\|u\|_{\hat{H}^{2}(\Omega)} \\
& \leq C h_{\mu}^{m}\|u\|_{\hat{H}^{m+1}(\Omega)}+C h_{\mu}^{m}\|u\|_{\hat{H}^{2}(\Omega)} \leq C h_{\mu}^{m}\|u\|_{\hat{H}^{m+1}(\Omega)} .
\end{aligned}
$$

From Lemma 1.6 and Assumption 1.3, then

$$
\begin{aligned}
\left\|u-u_{\mu}\right\|_{\hat{H}^{1}(\Omega)}^{2} & \leq C\left(\left|u-u_{\mu}\right|_{\hat{H}^{1}(\Omega)}^{2}+\left\|\left|u_{\mu}\right|\right\|\right) \\
& \leq C h_{\mu}^{m}\|u\|_{\hat{H}^{m+1}(\Omega)}+C h_{\mu}^{m}\left\|u_{\mu}\right\|_{\hat{H}^{1}(\Omega)} \\
& \leq C h_{\mu}^{m}\|u\|_{\hat{H}^{m+1}(\Omega)} \leq C_{1} h_{\mu}^{m}\|f\|_{\hat{H}^{m-1}(\Omega)} .
\end{aligned}
$$

The proof is now complete.

We therefore obtain a quasi-optimal rate of convergence for the approximate solution $u_{\mu} \in S_{\mu}$.

2. GFE spaces. In this section, we discuss the construction of a sequence of GFE spaces $S_{\mu}$ that satisfies Assumptions 1.3 and 1.4. Our choice of the sequence $S_{\mu}$ is determined by a sequence of data $\Sigma=\left\{\Omega_{j}, \phi_{j}, \Psi_{j}, \omega_{j}^{\star}\right\}$, described next. Later in this section (see Subsection 2.2), we recast the assumptions on the spaces $S_{\mu}$ as conditions, specifically Condition $\mathbf{A}\left(h_{\mu}\right), \mathbf{B}, \mathbf{C}$, and $\mathbf{D}$ below, on the data.

We follow the notations and basic techniques from [6] and [7]. 
2.1. Definition of GFE spaces. We begin by recalling the properties of the partition of unity on which the GFE spaces are based.

DEFINITION 2.1. Let $\Omega \subset \mathbb{R}^{n}$ be open set and $\left\{\omega_{j}\right\}_{j=1}^{N}$ be an open cover of $\Omega$ such that any $x \in \Omega$ belongs to at most $\kappa$ of the sets $\omega_{j}$. The covering sets $\left\{\omega_{j}\right\}$ are called patches.

Let $\left\{\phi_{j}\right\}$ be a partition of unity consisting of $W^{m, \infty}(\Omega)$ functions subordinated to the covering $\left\{\omega_{j}\right\}$. That is,

(i) $\operatorname{supp}\left(\phi_{j}\right) \subset \overline{\omega_{j}}$, and

(ii) $\sum_{j=1}^{N} \phi_{j}=1$, in $\Omega$.

The collection $\left\{\phi_{j}\right\}$ is then called a $\left(\kappa, C_{0}, C_{1}, \ldots, C_{m}\right)$ partition of unity, if

$$
\left\|\partial^{\alpha} \phi_{j}\right\|_{L^{\infty}}(\Omega) \leq C_{k} /\left(\operatorname{diam}\left(\omega_{j}\right)\right)^{k}, k=|\alpha| \leq m,
$$

for any $j=1, \ldots, N$.

We will also need the following basic concept (see e.g. [15]).

Definition 2.2. A set $\omega$ is star-shaped with respect to $\omega^{\star} \subset \omega$ if, for every $x \in \omega$ and every $y \in \omega^{\star}$, the segment with end points $x$ and $y$ is completely contained in $\omega$.

We next assume that we are given linear subspaces $\Psi_{j} \subset H^{m+1}\left(\omega_{j}\right)$ if $\omega_{j}$ does not touch the interface, and $\Psi_{j} \subset \hat{H}^{m+1}\left(\omega_{j}\right)$ if $\omega_{j}$ does touch the interface, for $j=1, \ldots N$. The space $\left\{\Psi_{j}\right\}$ are called local approximation spaces and they will be used to define the GFE space

$$
S=S_{G F E}:=\left\{w=\sum_{j=1}^{N} \phi_{j} v_{j}, v_{j} \in \Psi_{j}\right\} \subset \hat{H}^{1}(\Omega) .
$$

We will take the patches $\omega_{j}$ to be star-shaped with respect to given $\omega_{j}^{*}$, to be chosen later. Then, the set $\left\{\Omega_{j}, \phi_{j}, \Psi_{j}, \omega_{j}^{\star}\right\}$ will be called the set of data defining the GFE space $S$.

REMARK 2.3. The usual piecewise-linear hat functions satisfy the conditions of $a\left(\kappa, C_{0}, C_{1}\right)$ partition of unity (Definition 2.1). A more general choice of a partition of unity is given by the following well-known procedure. Let $\left\{\psi_{j}\right\}$ be a collection of functions which are supported on the patches $\left\{\omega_{j}\right\}$. Then setting $\phi_{j}:=\frac{\psi_{j}}{\sum_{j} \psi_{j}}$ yields a partition of unity, the elements of which are called Shepard functions. In practice, in order to solve Equation (1.4), the basis for the test and trial spaces needs to be found. One difficulty in finding a basis for the trial spaces is that, if $\left\{v_{j}\right\}$ is a basis of functions of the local spaces $\Psi_{j}$, the functions $\left\{\phi_{j} v_{i}\right\}$ may be linearly dependent or nearly linearly dependent. One can avoid the linear dependence if we use a "flattop" partition of unity (we refer the reader to [3, 5].) Our Condition $\mathbf{C}$ in the next subsection guarantees this linear independence.

We shall need the following standard Lemma (see for example [5]).

LEMma 2.4. Let $\left\{\psi_{j}\right\}$ be a countable collection of measurable functions defined on an open set $W$ and fix $s \geq 0$. Assume that there exists an integer $\kappa$ such that any point $x \in W$ can belong to no more than $\kappa$ of the sets $\operatorname{supp}\left(\psi_{j}\right)$. Let $f=\sum_{j} \psi_{j}$. Then there exists a constant $C>0$, depending only on $\kappa$, such that $\|f\|_{H^{s}(W)}^{2} \leq$ $C \sum_{j}\left\|\psi_{j}\right\|_{H^{s}(W)}^{2}$.

2.2. Conditions on GFE data defining $S_{\mu}$. In this subsection we introduce some conditions on the data defining a GFE space. Let $\tilde{h}$ be a constant that is small enough. 
For the definition of the local approximation spaces near the interface $\Gamma$ or the boundary $\partial \Omega$, we shall need the following construction. For each $j$ such that $\overline{\omega_{j}} \cap \partial \Omega \neq$ $\emptyset$, let us choose $x_{j} \in \overline{\omega_{j}} \cap \partial \Omega$ and define local coordinates near $x_{j}$ such that $x_{j}$ is mapped to $0 \in \mathbb{R}^{n}$ and the tangent space to $\partial \Omega$ at $x_{j}$ is mapped to $\left\{x_{n}=0\right\}=\mathbb{R}^{n-1}$. For $\tilde{h}$ small, if the diameter of $\omega_{j}$ is less than $\tilde{h}$, we can assume that $\overline{\omega_{j}} \cap \partial \Omega$ is contained in the graph of a smooth function $g_{j}: \mathbb{R}^{n-1} \rightarrow \mathbb{R}$. In the special coordinates chosen above, we denote $\bar{x}=\left(x_{1}, x_{2}, \cdots, x_{n-1}\right) \in \mathbb{R}^{n-1}$, so that $x=\left(\bar{x}, x_{n}\right) \in \mathbb{R}^{d}$. Denote by $d_{j}$ the diameter of $\omega_{j}$ and let $q_{j}: \mathbb{R}^{n-1} \rightarrow \mathbb{R}$ be a polynomial of order $m$ such that

$$
\left|g_{j}(\bar{x})-q_{j}(\bar{x})\right| \leq C d_{j}^{m+1}, \quad\left|\nabla g_{j}-\nabla q_{j}\right| \leq C d_{j}^{m},
$$

for all $\left(\bar{x}, x_{n}\right) \in \omega_{j}$. We can choose the function $q_{j}$ such that $\partial^{\alpha} q_{j}(0)=\partial^{\alpha} g_{j}(0)$, for all $|\alpha| \leq m$. We then denote by $\tilde{q_{j}}: \mathbb{R}^{n} \rightarrow \mathbb{R}^{d}$ the bijective map

$$
\tilde{q_{j}}(x)=\tilde{q_{j}}\left(\bar{x}, x_{n}\right)=\left(\bar{x}, x_{n}+q_{j}(\bar{x})\right) .
$$

The existence of such function $q_{j}$ follows by Taylor expanding the local boundary distance function. For example, let $\Omega$ be the unit disk in $\mathbb{R}^{2}$. We choose $m=1$ and local coordinates $(x, y)$ at the point $x_{0}=(1,0)$, We clearly have $g_{j}(x)=1-\sqrt{1-x^{2}}$ for $0 \leq x \leq d_{j}$. Then, we can choose $q_{j}(x)=x$, where $0 \leq x \leq d_{j}$. By Taylor expanding $g_{j}$ in local coordinates $g_{j}(x)=q_{j}(x)+\frac{g_{j}^{\prime \prime}(0)}{2} x^{2}+\cdots$, so that $\left|g_{j}(x)-q_{j}(x)\right| \leq$ $C d_{j}^{2}$. Similarly, $\left|\nabla g_{j}-\nabla q_{j}\right| \leq C d_{j}$ also holds.

We also choose a point $x_{i} \in \omega_{i} \cap \Gamma$, if this intersection is not empty. We then again choose local coordinates such that $x_{i}$ is mapped to 0 and the tangent space to $\Gamma$ at $x_{i}$ is mapped to $\mathbb{R}^{n-1}=\left\{x_{n}=0\right\}$. For $\tilde{h}$ small, we can assume that $\Gamma \cap \omega_{i}$ is contained in the graph of a smooth function $f_{i}: \mathbb{R}^{n-1} \rightarrow \mathbb{R}$. Let $r_{i}: \mathbb{R}^{n-1} \rightarrow \mathbb{R}$ be a polynomial of order $m$ satisfying:

$$
\left|f_{i}(\bar{x})-r_{i}(\bar{x})\right| \leq C d_{i}^{m+1}, \quad\left|\nabla f_{i}-\nabla r_{i}\right| \leq C d_{i}^{m},
$$

for all $\left(\bar{x}, x_{n}\right) \in \omega_{j}$. We also denote by $\tilde{r_{i}}: \mathbb{R}^{n} \rightarrow \mathbb{R}^{n}$ the map

$$
\tilde{r_{i}}(x):=\tilde{r_{i}}\left(\bar{x}, x_{n}\right)=\left(\bar{x}, x_{n}+r_{i}(\bar{x})\right),
$$

and set $\omega_{j} \backslash \Gamma=\omega_{j}^{+} \cup \omega_{j}^{-}$to be the decomposition of $\omega_{j}$ defined by the interface $\Gamma$.

An example of functions $r_{j}$ satisfying (2.3) can be constructed in the same way as for the case of the disk described above (take. e.g. $\Omega$ to be the unit disk and $\Gamma$ to be a smaller concentric circle to the unit circle). We also remark that, since $\tilde{q}_{j}^{-1}(x)=\left(\bar{x}, x_{n}-q_{j}(\bar{x})\right)$ and similarly for $\tilde{r}_{j}^{-1}$, both inverse maps are still polynomial in local coordinates at $x_{j}$.

Condition $\mathbf{A}(h)$ : We have that $\bar{\Omega}=\cup_{j=1}^{N} \bar{\omega}_{j}$. The set $\omega_{j}$ is open cover (patch) of diameter $d_{j} \leq h<1$ and $\omega_{j}^{\star} \subset \omega_{j}$ is an open ball of diameter $\geq \sigma d_{j}$ such that $\omega_{j}$ is star-shaped with respect to $\omega_{j}^{\star}$. If $\Gamma \cap \omega_{j} \neq \emptyset$, we require that $\Gamma$ divides $\omega_{j}$ into two subsets $\omega_{j}^{+}$and $\omega_{j}^{-}$, each of which is star-shaped with respect to a (possibly different) ball of diameter $\geq \sigma d_{j}$ contained in $\omega_{j}^{\star}$.

Condition B: The family of functions $\left\{\phi_{j}\right\}_{j=1}^{N}$ is a $\left(\kappa, C_{0}, C_{1}, \ldots, C_{m}\right)$ partition of unity (see Definition 2.1).

Condition C: We have $\phi_{j}=1$ on $\omega_{j}^{\star}$ for all $j=1, \ldots, N$ for which $\overline{\omega_{j}} \cap \partial \Omega \neq \emptyset$. 
The following condition defines the local approximation spaces $\Psi_{j}$. Let us denote by $\mathcal{P}_{m}$ the space of polynomials of order at most $m$ in $n$ variables.

\section{Condition D:}

(i) We have $\Psi_{j}=\mathcal{P}_{m}$ if $\overline{\omega_{j}} \cap \partial \Omega=\emptyset$ and $\overline{\omega_{j}} \cap \Gamma=\emptyset$.

(ii) If $\overline{\omega_{j}} \cap \partial \Omega \neq \emptyset$ and $\overline{\omega_{j}} \cap \Gamma=\emptyset$, then $\Psi_{j}$ consists of function $w$ of the form

$$
w(x)=\left(p \circ \tilde{q}_{j}^{-1}\right)(x), \quad p \in \mathcal{P}_{m}, \text { such that } p(\bar{x}, 0)=0 .
$$

(iii) If $\overline{\omega_{j}} \cap \Gamma \neq \emptyset$, then $\Psi_{j}$ consists of functions $w$ of the form

$$
w(x)= \begin{cases}p(x)+\left(p^{+} \circ{\tilde{r_{j}}}^{-1}\right)(x) & \text { in } \omega_{j}^{+}, \\ p(x) & \text { in } \omega_{j}^{-},\end{cases}
$$

where $p, p^{+} \in \mathcal{P}_{m}$ and $p^{+}(\bar{x}, 0)=0$.

REMARK 2.5. An equivalent form for the condition $p \in \mathcal{P}_{m}, p(\bar{x}, 0)=0$ in part (ii) above is that $p=x_{n} p_{1}, p_{1} \in \mathcal{P}_{m-1}$. Since $\tilde{q}_{j}^{-1}(x)=\left(\bar{x}, x_{n}-q_{j}(\bar{x})\right)$, we obtain $w\left(\bar{x}, x_{n}\right)=\left(x_{n}-q_{j}(\bar{x})\right)\left(p_{1} \circ \tilde{q}_{j}^{-1}\right)(x)$. It is not difficult to see that $\Psi_{j}$ is a subset of the space of polynomials in (ii). We observe that $p \circ \tilde{q}_{j}^{-1}$ is zero only across the approximate boundary defined by $r_{j}$, so the elements of $\Psi_{j}$ are "nearly zero" across the boundary $\partial \Omega$.

REMARK 2.6. The definition of $w$ in (2.6) is equivalent to

$$
w(x)= \begin{cases}p(x)+\left(x_{n}-r_{j}(\bar{x})\right)\left(p_{1} \circ \tilde{r}_{j}^{-1}\right)(x), & \text { in } \omega_{j}^{+}, \\ p(x) & \text { in } \omega_{j}^{-},\end{cases}
$$

where $p_{1} \in \mathcal{P}^{m-1}$. It is not difficult to see that $\Psi_{j}$ is a subset of the space of piecewise polynomials in (iii). We observe that $p^{+} \circ \tilde{r}_{j}^{-1}$ is zero only across the approximate interface defined by $r_{j}$, and not $\Gamma$. So $w$ jumps across $\Gamma$ and the elements of $\Psi_{j}$ are "nearly continuous" across the interface $\Gamma$, may have jump discontinuities in the derivatives across $\Gamma$, such that globally $\Psi_{j} \in \hat{H}^{m+1}(\Omega)$.

Condition $\mathbf{D}$ implies the following property:

Lemma 2.7. Assume $\mathbf{D}$ is satisfied. Let $0 \leq r \leq m+1$ be fixed. There exists a constant $C>0$, depending only on $\sigma, m, n$ and $r$, such that, for any patch $\overline{\omega_{j}} \cap \Gamma=\emptyset$, for any ball $\omega^{\star} \subset \omega_{j}$ of diameter $\geq \sigma d_{j}$, and for any $w \in \Psi_{j}$, we have

$$
\|w\|_{H^{r}\left(\omega_{j}\right)} \leq C\|w\|_{H^{r}\left(\omega^{\star}\right)} .
$$

Similarly, let $\bar{\omega}_{j}=\bar{\omega}^{+} \cup \bar{\omega}^{-}$be the decomposition of $\omega_{j}$ defined by $\Gamma$. Then there exists a constant $C>0$ such that for any $\overline{\omega_{j}} \cap \Gamma \neq \emptyset$, any $w \in \Psi_{j}$, any ball $\omega^{+\star} \subset \omega_{j}^{+}$of diameter $\geq \sigma d_{j}$, and any ball $\omega^{-\star} \subset \omega_{j}^{-}$of diameter $\geq \sigma d_{j}$, we have

$$
\left\|w^{+}\right\|_{H^{r}\left(\omega_{j}^{+}\right)}+\left\|w^{-}\right\|_{H^{r}\left(\omega_{j}^{-}\right)} \leq C\left(\left\|w^{+}\right\|_{H^{r}\left(\omega^{+\star}\right)}+\left\|w^{-}\right\|_{H^{r}\left(\omega^{-\star}\right)}\right) .
$$

Proof. Let $x_{0}, a$ be the center and radius of $\omega^{\star}$, so $\sigma d_{j} \leq a \leq d_{j} / 2$. We can assume $x_{0}=0$. Then $B\left(0, d_{j}\right)$ is a ball which contains $\omega_{j}$. We notice that, since $w$ is a polynomial, it extends to a smooth function on $B\left(0, d_{j}\right)$. If $w \in \mathcal{P}_{m}$, then

$$
\begin{aligned}
\|w\|_{H^{r}\left(\omega_{j}\right)}^{2} & \leq C \int_{B\left(0, d_{j}\right)} \sum_{\alpha \leq r}\left|\partial^{\alpha} w\right|^{2}(t) d t \\
& \leq\left(\frac{d_{j}}{a}\right) C \int_{\omega^{\star}} \sum_{\alpha \leq r}\left|\partial^{\alpha} w\right|^{2}\left(\frac{d_{j}}{a} x\right) d x \leq C\|w\|_{H^{r}\left(\omega^{\star}\right)}^{2},
\end{aligned}
$$


where $C$ only depends on $\sigma, m, n$, and $r$. Here, we recall, $m$ is a fixed integer and $n$ is the dimension of space. Above, we have used a change of variables and the fact that $w$ is a polynomial.

When $\omega_{j}$ intersects the interface $\Gamma$, we can derive similar inequalities in each subdomain $\omega_{j}^{+}$, and $\omega_{j}^{-}$owing to the Condition $\mathbf{A}(h)$ and the fact that $w$ is a piecewise polynomial across the interface $\Gamma$ in $\omega_{j}$. The proof is now complete.

2.3. Inverse inequalities. We will need the following inverse inequality (see for example [6]).

Lemma 2.8 (Inverse Inequality). There exists $C>0$, depending only on $r, s$, and $m$, such that

$$
\|p\|_{H^{s}\left(\omega_{j}\right)} \leq C\left(d_{j}\right)^{r-s}\|p\|_{H^{r}\left(\omega_{j}\right)}
$$

for all $0 \leq r \leq s \leq m$, all $j \in \mathbb{Z}_{+}$, and all polynomials $p$ of degree $\leq m$, where $d_{j}=\operatorname{diam}\left(\omega_{j}\right)$.

From this lemma and Condition $\mathbf{A}(h)$, we immediately obtain the following generalization.

Lemma 2.9. There exists $C>0$, depending only on $r$, $s$, and $m$, such that

$$
\|p\|_{H^{s}\left(\omega_{j}^{ \pm}\right)} \leq C\left(d_{j}\right)^{r-s}\|p\|_{H^{r}\left(\omega_{j}^{ \pm}\right)}
$$

for any function $p: \omega_{j} \rightarrow \mathbb{R}$ that is given by a polynomial of degree $\leq m$ on each side $\omega_{j}^{ \pm}$of the interface $\Gamma$ and for any $j \in \mathbb{Z}_{+}$, and $0 \leq r \leq s \leq m$.

2.4. Conditions on the sequence of GFE data defining $S_{\mu}$. Our goal is to define a sequence of data sets $\Sigma_{\mu}=\left\{\omega_{j}^{\mu}, \phi_{j}^{\mu}, \Psi_{j}^{\mu}, \omega_{j}^{\star \mu}\right\}$, yielding GFE-spaces $S_{\mu}$, as in the previous Subsection 2.1, by

$$
S_{\mu}=\left\{\sum_{j}^{N_{\mu}} \phi_{j}^{\mu} v_{j}, v_{j} \in \Psi_{j}^{\mu}\right\} \subset \hat{H}^{1}(\Omega) .
$$

Let us denote by $h_{\mu}$ the maximum of the diameters of the patches $\omega_{j}^{\mu}$ defining $S_{\mu}$. Also, let $\tilde{h}=\max h_{\mu}$. Note that by assuming $h_{\mu} \rightarrow 0$ and by possibly excluding the first few spaces $S_{\mu}$ in the sequence we can assume that $\tilde{h}$ is sufficiently small. For notational convenience, whenever $\mu$ is fixed, we drop it from the notation in what follows.

We are ready now to introduce the conditions on the sequence of data $\Sigma_{\mu}$ used to define the flat top GFE spaces $S_{\mu}$. Namely, we assume that there exist constants $C_{j}, \sigma, \kappa$ which will be called structural constants and a sequence $h_{\mu} \rightarrow 0$, as $\mu \rightarrow \infty$, such that, for any $\mu, \Sigma_{\mu}$ satisfies Conditions $\mathbf{A}\left(h_{\mu}\right), \mathbf{B}, \mathbf{C}$, and $\mathbf{D}$. (In particular, $d_{j}^{\mu} \leq h_{\mu}$, with $d_{j}^{\mu}=\operatorname{diam}\left(\omega_{j}^{\mu}\right)$.) Note that if we denote, as before, by $N_{\mu}$ the number of elements of the $\mu$ th partition of unity, then $N_{\mu} \rightarrow \infty$ as $\mu \rightarrow \infty$.

Flat top GFE spaces were considered before by $[3,21]$ and in other papers. The conditioning of the resulting linear system was studied in [25]. Let us choose a basis $v_{j}^{\mu}$ of $\Psi_{j}^{\mu}$ and consider $\left\{v_{j}^{\mu} \phi_{j}^{\mu}\right\}$, which is a system of generators of $S_{\mu}$. In general, this system will not form a basis of $S_{\mu}$, and if we define the stiffness matrix using this system, the resulting system will be singular. The matrix may not be singular if exact integration is used, but become singular due to errors arising from numerical integration. More details can be founded in [1, 21, 20]. 
3. Properties of the GFE spaces $S_{\mu}$. The main result of this section is that a sequence $S_{\mu}$ of GFE spaces obtained from data satisfying Conditions $\mathbf{A}\left(h_{\mu}\right), \mathbf{B}, \mathbf{C}$, and $\mathbf{D}$ given in the previous section also satisfies Assumptions 1.3 and 1.4 of Section 2. Whenever no confusion can arise, we will omit the explicit dependence on $\mu$. We also assume throughout that $h_{\mu}$ is chosen sufficiently small.

We recall the functions $g_{j}, q_{j}, f_{i}$, and $r_{i}: \mathbb{R}^{n-1} \rightarrow \mathbb{R}$ defined in the previous section. Let $\tilde{q_{j}}, \tilde{r_{i}}(x)$ be defined by equation $(2.2),(2.4)$, and similarly define

$$
\begin{gathered}
\tilde{g}_{j}(x)=\tilde{g}_{j}\left(\bar{x}, x_{n}\right)=\left(\bar{x}, x_{n}+g_{j}(\bar{x})\right), \\
\tilde{f}_{i}(x)=\tilde{f}_{i}\left(\bar{x}, x_{n}\right)=\left(\bar{x}, x_{n}+f_{i}(\bar{x})\right) .
\end{gathered}
$$

We then have

$$
\begin{array}{ll}
{\tilde{g_{j}}}^{-1}(x)=\left(\bar{x}, x_{n}-g_{j}(\bar{x})\right), \quad \tilde{f}_{i}^{-1}(x)=\left(\bar{x}, x_{n}-f_{i}(\bar{x})\right), \\
{\tilde{q_{j}}}^{-1}(x)=\left(\bar{x}, x_{n}-q_{j}(\bar{x})\right), \quad{\tilde{r_{i}}}^{-1}(x)=\left(\bar{x}, x_{n}-r_{i}(\bar{x})\right) .
\end{array}
$$

We will need the following result from [6].

Lemma 3.1. There exists a constant $C>0$ depending only on the structural constants such that for any $j$ satisfying $\overline{\omega_{j}} \cap \partial \Omega \neq \emptyset$ and any polynomial $p$ of degree $m$, we have

$$
\begin{gathered}
\left\|p \circ{\tilde{g_{j}}}^{-1}-p \circ{\tilde{q_{j}}}^{-1}\right\|_{L^{2}\left(\omega_{j}\right)} \leq C d_{j}^{m+1}\|p\|_{H^{1}\left(\omega_{j}\right)}, \quad \text { and } \\
\left\|p \circ{\tilde{g_{j}}}^{-1}-p \circ{\tilde{q_{j}}}^{-1}\right\|_{H^{1}\left(\omega_{j}\right)} \leq C d_{j}^{m}\|p\|_{H^{1}\left(\omega_{j}\right)} .
\end{gathered}
$$

Similarly, we have the following result.

Lemma 3.2. There exists a constant $C>0$ depending only on the structural constants such that for each $i$ satisfying $\overline{\omega_{i}} \cap \Gamma \neq \emptyset$ and any $p$ which is a polynomial of degree $m$ in $\omega_{i}^{+}$, we have

$$
\begin{gathered}
\left\|p \circ{\tilde{f_{i}}}^{-1}-p \circ{\tilde{r_{i}}}^{-1}\right\|_{L^{2}\left(\omega_{i}^{+}\right)} \leq C d_{i}^{m+1}\|p\|_{H^{1}\left(\omega_{i}^{+}\right)}, \quad \text { and } \\
\left\|p \circ \tilde{f}_{i}^{-1}-p \circ{\tilde{r_{i}}}^{-1}\right\|_{H^{1}\left(\omega_{i}^{+}\right)} \leq C d_{i}^{m}\|p\|_{H^{1}\left(\omega_{i}^{+}\right)} .
\end{gathered}
$$

These two inequalities also hold in $\omega_{i}^{-}$.

Proof. We apply Lemma 3.1 to $p$ on $\omega_{i}^{+}$, which is possible since $\omega_{i} \subset \Omega$, in other words, in the above lemma $\omega_{i}$ is on one side of the boundary, in the same way as $\omega_{i}^{ \pm}$ is on one side of $\Gamma$. We then proceed similarly in $\omega_{i}^{-}$. $\square$

These lemmas give the following corollaries, the first near the boundary $\partial \Omega$, the second across the interface $\Gamma$. (For a definition of the Sobolev spaces $H^{s}(\partial \Omega), s \in \mathbb{R}$, and their associated norm, we refer for example to [39].)

Corollary 3.3. Let $p \in \mathcal{P}_{m}$, then for any $j$ such that $\bar{\omega}_{j} \cap \partial \Omega \neq \emptyset$, it holds

$$
\left\|\phi_{j}\left(p \circ \tilde{g}_{j}^{-1}-p \circ \tilde{q}_{j}^{-1}\right)\right\|_{H^{1}\left(\omega_{j}\right)} \leq C d_{j}^{m}\|p\|_{H^{1}\left(\omega_{j}\right)} .
$$

If in addition $p \in \mathcal{P}_{m}$ vanishes on $\left\{x_{n}=0\right\}$, we then have

$$
\left\|\phi_{j}\left(p \circ \tilde{q}_{j}^{-1}\right)\right\|_{H^{1 / 2}(\partial \Omega)} \leq C d_{j}^{m}\|p\|_{H^{1}\left(\omega_{j}^{\star}\right)} .
$$

Above $C$ is a constant independent of $p, \mu$, and $j$, but dependent on $m$. 
Proof. The first inequality follows directly from Lemma 3.1. The second inequality is proven in the same way as in [6], given that it is a local property near the boundary. $\square$

Corollary 3.4. Let $\omega_{j} \cap \Gamma \neq \emptyset$ and let $p: \omega_{j} \rightarrow \mathbb{R}$ be given by a piecewise polynomial function that equals a polynomial on each side of the interface $\Gamma$. Then, there exits a constant $C$ independent of $p, \mu$, and $j$, but dependent on the structural constants, such that

$$
\left\|\phi_{j}\left(p \circ \tilde{r}_{j}^{-1}-p \circ \tilde{f}_{j}^{-1}\right)\right\|_{\hat{H}^{1}\left(\omega_{j}\right)} \leq C d_{j}^{m}\|p\|_{\hat{H}^{1}\left(\omega_{j}\right)} .
$$

If in addition $p$ vanishes on $\left\{x_{n}=0\right\}$, we then have

$$
\left\|\phi_{j}\left(p \circ \tilde{r}_{j}^{-1}\right)\right\|_{H^{1 / 2}\left(\Gamma \cap \omega_{j}\right)} \leq C d_{j}^{m}\|p\|_{\hat{H}^{1}\left(\omega_{j}^{\star}\right)}
$$

We remark that in the above corollary $p$ may change patch by patch along $\Gamma$.

Proof. Using Lemma 3.2, Condition B or Definition 2.1, gives

$$
\begin{aligned}
& \left\|\phi_{j}\left(p \circ \tilde{r}_{j}^{-1}-p \circ \tilde{f}_{j}^{-1}\right)\right\|_{H^{1}\left(\omega_{j}^{+}\right)} \leq\left\|\phi_{j}\right\|_{L^{\infty}\left(\omega_{j}^{+}\right)}\left\|p \circ \tilde{r}_{j}^{-1}-p \circ \tilde{f}_{j}^{-1}\right\|_{H^{1}\left(\omega_{j}^{+}\right)} \\
& +\sum_{i=1}^{n}\left\|\partial_{i} \phi_{j}\right\|_{L^{\infty}\left(\omega_{j}^{+}\right)}\left\|p \circ \tilde{r}_{j}^{-1}-p \circ \tilde{f}_{j}^{-1}\right\|_{L^{2}\left(\omega_{j}^{+}\right)} \\
& \quad \leq C d_{j}^{m}\|p\|_{H^{1}\left(\omega_{j}^{+}\right)}+C d_{j}^{-1} d_{j}^{m+1}\|p\|_{H^{1}\left(\omega_{j}^{+}\right)} \leq C d_{j}^{m}\|p\|_{H^{1}\left(\omega_{j}^{+}\right)} .
\end{aligned}
$$

Since a similar estimate holds in $\omega_{j}^{-}$, we obtain the first inequality in the broken Sobolev space $\hat{H}^{1}\left(\omega_{j}\right)$.

We next observe that the hypotheses on $\Gamma$ and $\partial \Omega$ imply that we can extend any function in $H^{m}\left(\Omega_{k}\right), k=1, \ldots, K$, where $\Omega_{k}$ are as in (0.3), to a function in $H^{m}(\Omega)$ with control on the norm in terms only of the geometry of $\Gamma$ and $\Omega$.

Then, if $p$ vanishes on $\left\{x_{n}=0\right\}$, we have $\phi_{j}\left(p \circ \tilde{f}_{j}^{-1}\right)=0$, and consequently

$$
\begin{aligned}
& \left\|\phi_{j}\left(p \circ \tilde{r}_{j}^{-1}\right)\right\|_{H^{1 / 2}\left(\Gamma \cap \omega_{j}\right)}=\left\|\phi_{j}\left(p \circ \tilde{r}_{j}^{-1}-p \circ \tilde{f}_{j}^{-1}\right)\right\|_{H^{1 / 2}\left(\Gamma \cap \omega_{j}\right)} \\
& \quad \leq\left\|\phi_{j}\left(p \circ \tilde{r}_{j}^{-1}-p \circ \tilde{f}_{j}^{-1}\right)\right\|_{H^{1 / 2}(\Gamma)} \leq C\left\|\phi_{j}\left(p \circ \tilde{r}_{j}^{-1}-p \circ \tilde{f}_{j}^{-1}\right)\right\|_{\hat{H}^{1}(\Omega)} \\
& \quad \leq C\left\|\phi_{j}\left(p \circ \tilde{r}_{j}^{-1}-p \circ \tilde{f}_{j}^{-1}\right)\right\|_{\hat{H}^{1}\left(\omega_{j}\right)} \leq C d_{j}^{m}\|p\|_{\hat{H}^{1}\left(\omega_{j}\right)} \leq C d_{j}^{m}\|p\|_{\hat{H}^{1}\left(\omega_{j}^{\star}\right)}
\end{aligned}
$$

where we used the trace theorem on each side of the interface and Lemma 2.7. By construction $C$ above depends only on the trace constant on the $\Omega_{k}$, and the structural constants by Condition B.

Now we are ready to prove that our first assumption, that is nearly zero boundary conditions and interface matching, is satisfied by the sequence of GFE-space $S_{\mu}$ introduced in Section 2.

Proposition 3.5. Let $S_{\mu} \subset \hat{H}^{1}(\Omega)$ be the sequence of GFE spaces defined by the data $\Sigma_{\mu}$ satisfying conditions $A\left(h_{\mu}\right), B, C$ and $D$. Then the sequence $S_{\mu}$ satisfies Assumption 1.3.

Proof. We first show that elements in $S_{\mu}$ have nearly zero boundary values. Let $v_{j} \in \Psi_{j}^{\mu}$ and $w=\sum \phi_{j} v_{j} \in S_{\mu}$. We may assume $v_{j}=0$ if $\bar{\omega}_{j} \cap \partial \Omega=\emptyset$. Then 
$v_{j}=p_{j} \circ{\tilde{q_{j}}}^{-1}$, for some $p_{j} \in \mathcal{P}_{m}$ vanishing on $\mathbb{R}^{n-1}$ (hence $p_{j} \circ \tilde{g}_{j}^{-1}=0$.) Therefore,

$$
\begin{aligned}
\|w\|_{L^{2}(\partial \Omega)}^{2} & \leq C \sum_{j}\left\|\phi_{j} v_{j}\right\|_{L^{2}(\partial \Omega)}^{2}=C \sum_{j}\left\|\phi_{j}\left(p_{j} \circ \tilde{q}_{j}^{-1}\right)\right\|_{L^{2}(\partial \Omega)}^{2} \\
& \leq C \sum_{j} d_{j}^{2 m}\left\|p_{j}\right\|_{H^{1}\left(\omega_{j}\right)}^{2} \leq C h_{\mu}^{2 m} \sum_{j}\left\|p_{j}\right\|_{H^{1}\left(\omega_{j}^{\star}\right)}^{2} \leq C h_{\mu}^{2 m} \sum_{j}\left\|v_{j}\right\|_{H^{1}\left(\omega_{j}^{\star}\right)}^{2} .
\end{aligned}
$$

Here we have used Lemma 2.4 and Corollary 3.3. By Condition $\mathbf{C}, \phi_{j}^{\mu}=1$ on $\omega_{j}^{\star}$ and hence the sets $\omega_{j}^{\star}$ do not intersect. Therefore $\sum_{j}\left\|v_{j}\right\|_{H^{1}\left(\omega_{j}^{\star}\right)}^{2}=\|w\|_{H^{1}\left(\cup \omega_{j}^{\star}\right)}^{2}$ and

$$
\|w\|_{L^{2}(\partial \Omega)}^{2} \leq C h_{\mu}^{2 m}\|w\|_{H^{1}\left(\cup \omega_{j}^{\star}\right)}^{2} \leq C h_{\mu}^{2 m}\|w\|_{\hat{H}^{1}(\Omega)}^{2} .
$$

We now proceed to estimate the jump on the interface $\Gamma$ using a similar reasoning. Indeed, as before, let $v_{j} \in \Psi_{j}^{\mu}$ and let $w=\sum \phi_{j} v_{j} \in S_{\mu}$. We may assume again that $v_{j}=0$ if $\bar{\omega}_{j} \cap \Gamma=\emptyset$. Then,

$$
v_{j}=p_{j}+\left(p_{j}^{+} \circ{\tilde{q_{j}}}^{-1}\right) 1_{\omega_{j}^{+}},
$$

where $1_{\omega_{j}^{+}}$is the characteristic function of $\omega_{j}^{+}, p_{j}, p_{j}^{+} \in \mathcal{P}_{m}$, and $p_{j}^{+}$vanishes on $\mathbb{R}^{n-1}$ by Condition $\mathbf{D}$. Therefore,

$$
p_{j}^{+} \circ \tilde{f}_{j}^{-1}(x)=p_{j}^{+}\left(\bar{x}, x_{n}-f_{j}(\bar{x})\right)=0
$$

on $\Gamma$ and we obtain

$$
\begin{aligned}
\left\|w_{+}-w_{-}\right\|_{L^{2}(\Gamma)}^{2} & \leq \sum_{j}\left\|\phi_{j}\left(p_{j}^{+} \circ \tilde{r}_{j}^{-1}\right)\right\|_{L^{2}\left(\Gamma \cap \omega_{j}\right)}^{2} \\
& =\sum_{j}\left\|\phi_{j}\left(p_{j}^{+} \circ\left(\tilde{r}_{j}^{-1}-\tilde{f}_{j}^{-1}\right)\right)\right\|_{L^{2}\left(\Gamma \cap \omega_{j}\right)}^{2} \\
& \leq C \sum_{j} d_{j}^{2 m}\left\|p_{j}^{+}\right\|_{H^{1}\left(\tilde{f}^{-} 1\left(\omega_{j}^{\star}\right)\right)}^{2} \text { by Corollary } 3.4 \\
& \leq C h_{\mu}^{2 m}\|w\|_{\hat{H}^{1}(\Omega)}^{2},
\end{aligned}
$$

where the last step is proved as in the first part of the proof. The proof is now complete. $\mathrm{Q}$

Next we are going to prove that the sequence $S_{\mu}$ also satisfies our second assumption, that is, the assumption of approximability, using Lemmas 3.1 and 3.2 and the following result.

Lemma 3.6. (i) Let $\overline{\omega_{j}} \cap \Gamma=\emptyset$, and let $u \in H^{m+1}\left(\omega_{j}\right)$ satisfy $u=0$ on $\overline{\omega_{j}} \cap \partial \Omega$. Then there exists a polynomial $w \in \Psi_{j}^{\mu}$ such that

$$
\begin{aligned}
\|u-w\|_{H^{1}\left(\omega_{j}\right)} & \leq C d_{j}^{m}\|u\|_{H^{m+1}\left(\omega_{j}\right)}, \text { and } \\
\|u-w\|_{L^{2}\left(\omega_{j}\right)} & \leq C d_{j}^{m+1}\|u\|_{H^{m+1}\left(\omega_{j}\right)}
\end{aligned}
$$

for a constant $C$ independent of $u, \mu$, and $j$.

(ii) Let $\overline{\omega_{j}} \cap \Gamma \neq \emptyset$, and let $u \in \hat{H}^{m+1}\left(\omega_{i}\right) \cap H^{1}(\Omega)$. Then there exists $w \in \Psi_{i}^{\mu}$ such that

$$
\begin{aligned}
\|u-w\|_{\hat{H}^{1}\left(\omega_{i}\right)} & \leq C d_{i}^{m}\|u\|_{\hat{H}^{m+1}\left(\omega_{i}\right)}, \text { and } \\
\|u-w\|_{L^{2}\left(\omega_{i}\right)} & \leq C d_{i}^{m+1}\|u\|_{\hat{H}^{m+1}\left(\omega_{i}\right)} .
\end{aligned}
$$


Proof. (i) Let $v=u \circ \tilde{g}_{j}=u\left(\bar{x}, x_{n}+g_{j}(\bar{x})\right)$. Since $\left.u\right|_{\partial \Omega}=u\left(\bar{x}, g_{j}(\bar{x})\right)=0$, we have that $v=0$ on $\left\{x_{n}=0\right\} \cap \tilde{g}_{j}^{-1}\left(\omega_{j}\right)$, where $\tilde{g}_{j}^{-1}(x)=\left(\bar{x}, x_{n}-g_{j}(\bar{x})\right)$. Without loss of generality, we may assume for $h_{\mu}$ small enough that $\tilde{g}_{j}^{-1}\left(\omega_{j}\right)$ lies on one side of $\mathbb{R}^{n-1}$, for example $U_{+}:=\tilde{g}_{j}^{-1}\left(\omega_{j}\right) \subset\left\{x, x_{n} \geq 0\right\}$. Let $U$ be the union of the closure of $\tilde{g}_{j}^{-1}\left(\omega_{j}\right)$ and of its symmetric subset with respect to $\mathbb{R}^{n-1}$. Define $v_{1} \in H^{1}(U)$ to be odd extension of $v$. and let $p_{1}$ be the $H^{1}(U)$ - projection of $v_{1}$ onto the subspace $\mathcal{P}_{m}$ of polynomials of degree $\leq m$. It is easy to see that $p_{1}$ is odd and $p_{1}=0$ on $\mathbb{R}^{n-1}$. From standard approximation results [15], it follows that

$$
\left\|v-p_{1}\right\|_{H^{1}\left(U_{+}\right)} \leq C d_{j}^{m}\|v\|_{H^{m+1}\left(U_{+}\right)} .
$$

Then,

$$
\left\|u-p_{1} \circ \tilde{g}_{j}^{-1}\right\|_{H^{1}\left(\omega_{j}\right)} \leq\left\|v-p_{1}\right\|_{H^{1}\left(U_{+}\right)} \leq C d_{j}^{m}\left\|v_{1}\right\|_{H^{m+1}\left(U_{+}\right)} \leq C d_{j}^{m}\|u\|_{H^{m+1}\left(\omega_{j}\right)} .
$$

Let $w=p_{1} \circ \tilde{q}_{j}^{-1}$, then $w \in \Psi_{j}^{\mu}$ and

$$
\begin{aligned}
\|u-w\|_{H^{1}\left(\omega_{j}\right)} \leq \| u- & p_{1} \circ \tilde{g}_{j}^{-1}\left\|_{H^{1}\left(\omega_{j}\right)}+\right\| p_{1} \circ \tilde{g}_{j}^{-1}-p_{1} \circ \tilde{q}_{j}^{-1} \|_{H^{1}\left(\omega_{j}\right)} \\
\leq & C d_{j}^{m}\|u\|_{H^{m+1}\left(\omega_{j}\right)}+C d_{j}^{m}\left\|p_{1}\right\|_{H^{1}\left(\omega_{j}\right)} \leq C d_{j}^{m}\|u\|_{H^{m+1}\left(\omega_{j}\right)},
\end{aligned}
$$

where we have used Lemma 3.1.

Since both $v_{1}$ and $p_{1}$ vanish for $x_{n}=0$, Poincaré's inequality gives

$$
\left\|v_{1}-p_{1}\right\|_{L^{2}\left(U_{+}\right)} \leq C d_{j}\left\|v_{1}-p_{1}\right\|_{H^{1}\left(U_{+}\right)} \leq C d_{j}^{m+1}\left\|v_{1}\right\|_{H^{m+1}\left(\omega_{j}\right)} .
$$

Hence,

$$
\begin{aligned}
&\|u-w\|_{L^{2}\left(\omega_{j}\right)} \leq \| u-p_{1} \circ \tilde{g}_{j}^{-1}\left\|_{L^{2}\left(\omega_{j}\right)}+\right\| p_{1} \circ \tilde{g}_{j}^{-1}-p_{1} \circ \tilde{q}_{j}^{-1} \|_{L^{2}\left(\omega_{j}\right)} \\
& \leq\left\|v_{1}-p_{1}\right\|_{L^{2}(U)}+C d_{j}^{m+1}\left\|p_{1}\right\|_{H^{1}\left(\omega_{j}\right)} \leq C d_{j}^{m+1}\|u\|_{H^{m+1}\left(\omega_{j}\right)} .
\end{aligned}
$$

This completes the proof of the first part of this lemma.

(ii) We now proceed to the proof of the second part. We assume that $\omega_{i}$ intersects the interface $\Gamma$. We know that $u \in \hat{H}^{m+1}\left(\omega_{i}\right)$. That is, $u \in H^{m+1}\left(\omega_{i}^{+}\right)$and $u \in H^{m+1}\left(\omega_{i}^{-}\right)$, by the definition of the broken Sobolev spaces $\hat{H}$. Standard extension theorems (see e.g. [39, pages 284-286] for more details). To see that the constants do not depend on $i$, we notice that we can embed each $\omega_{i}$ in a fixed ball with chosen point of the interface mapping to the origin. We can then apply a diffeomorphism that straightens the interface. As $\mu$ increases, this diffeomorphism approaches a linear transformation, which can be assumed to be an orthogonal transformation, thus preserving the Sobolev norm. We can then apply the extension theorem for a half space with uniform control on the Sobolev norm. The constant in our extension theorem for the sets $\omega_{i}^{ \pm} \subset \omega_{i}$ can then be chosen to be a multiple of the constant appearing in the extension for half-space. Alternatively, we can employ a global extension from one side of $\Gamma$ as in the proof of Lemma 3.4.

We now show that there exists $v \in H^{m+1}\left(\omega_{i}\right)$ such that $u=v$ on $\omega_{i}^{-}$and

$$
\|v\|_{H^{m+1}\left(\omega_{i}\right)} \leq C\|u\|_{H^{m+1}\left(\omega_{i}^{-}\right)} .
$$


By standard approximation results (see [15, Section 4.1], for example), there exists $p \in \mathcal{P}_{m}$ such that

$$
\begin{aligned}
\|v-p\|_{H^{k}\left(\omega_{i}\right)} \leq C d_{i}^{m+1-k} & \|v\|_{H^{m+1}\left(\omega_{i}\right)} \\
& \leq C d_{i}^{m+1-k}\|u\|_{H^{m+1}\left(\omega_{i}^{-}\right)} \leq C d_{i}^{m+1-k}\|u\|_{\hat{H}^{m+1}\left(\omega_{i}\right)}
\end{aligned}
$$

for $k=0,1, \ldots, m+1$.

We next define $v^{+}:=(u-v) \circ \tilde{f}_{i}$ on $\tilde{f}_{i}^{-1}\left(\omega_{i}\right)$. Since $u=v$ on $\omega_{i}^{-}, v^{+} \equiv 0$ on $\tilde{f}^{-1}\left(\bar{\omega}_{i}^{-}\right)$, and in particular on $\mathbb{R}^{n-1}$, we obtain

$$
\left\|v^{+}\right\|_{H^{m+1}\left(\tilde{f}_{i}^{-1}\left(\omega_{i}^{+}\right)\right)} \leq C\|u\|_{\hat{H}^{m+1}\left(\omega_{i}\right)},
$$

owing to (3.1).

We now proceed as in the proof of (i). Namely, let $p^{+}$be defined in the same way as $p_{1}$ was defined in the proof of (i), but using $v^{+}$instead of $v$. In particular, $p^{+}$is odd and hence $p^{+}=0$ on $\mathbb{R}^{n-1}$. As before,

$$
\left\|v^{+}-p^{+}\right\|_{H^{1}\left(\tilde{f}_{i}^{-1}\left(\omega_{i}^{+}\right)\right)} \leq C d_{i}^{m}\left\|v^{+}\right\|_{H^{m+1}\left(\tilde{f}_{i}^{-1}\left(\omega_{i}^{+}\right)\right)} .
$$

Then inequalities $3.1,3.2,3.3$, and 3.4 give

$$
\begin{gathered}
\left\|(u-p)-p^{+} \circ \tilde{f}_{i}^{-1}\right\|_{H^{1}\left(\omega_{i}^{+}\right)} \leq C\left(\left\|u-v-p^{+} \circ \tilde{f}_{i}^{-1}\right\|_{H^{1}\left(\omega_{i}^{+}\right)}+\|v-p\|_{H^{1}\left(\omega_{i}^{+}\right)}\right) \\
\leq C\left(\left\|v^{+}-p^{+}\right\|_{H^{1}\left(\tilde{f}_{i}^{-1}\left(\omega_{i}^{+}\right)\right)}+\|v-p\|_{H^{1}\left(\omega_{i}^{+}\right)}\right) \\
\leq C d_{i}^{m}\left\|v^{+}\right\|_{H^{m+1}\left(\tilde{f}_{i}^{-1}\left(\omega_{i}^{+}\right)\right)}+C d_{i}^{m}\|u\|_{\hat{H}^{m+1}\left(\omega_{i}\right)} \leq C d_{i}^{m}\|u\|_{\hat{H}^{m+1}\left(\omega_{i}\right)} .
\end{gathered}
$$

Next, let $w=p+p^{+} \circ \tilde{r}_{i}^{-1} 1_{\omega_{i}^{+}}$, where $1_{\omega_{i}^{+}}$is the characteristic function of the set $\omega_{i}^{+}$. Then

$$
\begin{aligned}
\|u-w\|_{H^{1}\left(\omega_{i}^{+}\right)} & \leq\left\|(u-p)-p^{+} \circ \tilde{f}_{i}^{-1}\right\|_{H^{1}\left(\omega_{i}^{+}\right)}+\left\|p^{+} \circ \tilde{f}_{i}^{-1}-p^{+} \circ \tilde{r}_{i}^{-1}\right\|_{H^{1}\left(\omega_{i}^{+}\right)} \\
& \leq C d_{i}^{m}\|u\|_{\hat{H}^{m+1}\left(\omega_{i}\right)}
\end{aligned}
$$

where we have also used Lemma 3.1 and the fact that

$$
\left\|p^{+}\right\|_{H^{1}\left(\tilde{f}_{i}^{-1}\left(\omega_{i}^{+}\right)\right)} \leq C\|u\|_{\hat{H}^{m+1}\left(\omega_{i}\right)} .
$$

This last inequality is true because

$$
\begin{aligned}
&\left\|p^{+}\right\|_{H^{1}\left(\tilde{f}_{i}^{-1}\left(\omega_{i}^{+}\right)\right)} \leq C\left\|p^{+}-v^{+}\right\|_{H^{1}\left(\tilde{f}_{i}^{-1}\left(\omega_{i}^{+}\right)\right)}+\left\|v^{+}\right\|_{H^{1}\left(\tilde{f}_{i}^{-1}\left(\omega_{i}^{+}\right)\right)} \\
& \leq\left\|v^{+}\right\|_{H^{m+1}\left(\left(\tilde{f}_{i}^{-1}\left(\omega_{i}^{+}\right)\right)\right)} \leq C\|u\|_{\hat{H}^{m+1}\left(\omega_{i}\right)}
\end{aligned}
$$

On the other side of the interface, we have instead

$$
\|u-w\|_{H^{1}\left(\omega_{i}^{-}\right)}=\|v-p\|_{H^{1}\left(\omega_{i}^{+}\right)} \leq C d_{i}^{m}\|u\|_{\hat{H}^{m}\left(\omega_{i}\right)},
$$

which together with the above calculations gives

$$
\|u-w\|_{\hat{H}^{1}\left(\omega_{i}\right)} \leq C d_{i}^{m}\|u\|_{\hat{H}^{m}\left(\omega_{i}\right)} .
$$

Finally, Poincaré's inequality again gives

$$
\left\|v-p^{+} \circ \tilde{f}^{-1}\right\|_{L^{2}\left(\omega_{i}\right)} \leq C d_{i}\left\|v_{1}-p^{+} \circ \tilde{f}^{-1}\right\|_{H^{1}\left(\omega_{i}\right)} \leq C d_{i}^{m+1}\left\|v_{1}\right\|_{H^{m+1}\left(\omega_{i}\right)} .
$$


Then

$$
\begin{aligned}
\|u-w\|_{L^{2}\left(\omega_{i}^{+}\right)} \leq & C\left\|(u-v)-p^{+} \circ \tilde{f}_{i}^{-1}\right\|_{L^{2}\left(\omega_{i}^{+}\right)}+\|v-p\|_{L^{2}\left(\omega_{i}^{+}\right)} \\
& \leq C\left(\left\|v_{1}-p^{+}\right\|_{L^{2}\left(\tilde{f}\left(\omega_{i}\right)\right)}+\|v-p\|_{L^{2}\left(\omega_{i}\right)}\right) \leq C d_{i}^{m+1}\|u\|_{\hat{H}^{m+1}\left(\omega_{i}\right)} .
\end{aligned}
$$

Since

$$
\|u-w\|_{L^{2}\left(\omega_{i}^{-}\right)}=\|v-p\|_{L^{2}\left(\omega_{i}^{-}\right)} \leq C d_{i}^{m+1}\|u\|_{\hat{H}^{m+1}\left(\omega_{i}\right)},
$$

we also have

$$
\|u-w\|_{L^{2}\left(\omega_{i}\right)} \leq C d_{i}^{m+1}\|u\|_{\hat{H}^{m+1}\left(\omega_{i}\right)} .
$$

The proof is now complete.

We are ready to prove that our second assumption, the assumption on approximability is satisfied.

Proposition 3.7. The sequence of GFEM spaces $S_{\mu}$ satisfies Assumption 1.4.

Proof. Let $u \in \hat{H}^{m+1}(\Omega) \cap H_{0}^{1}(\Omega)$. If $\overline{\omega_{j}}$ does not intersect $\partial \Omega$ and $\Gamma$, we define $w_{j} \in \Psi_{j}^{\mu}=\mathcal{P}_{m}$ to be the orthogonal projection of $u$ onto $\mathcal{P}_{m}$ in $H^{1}\left(\omega_{j}\right)$. Otherwise we define $w_{j} \in \Psi_{j}$ using Lemma 3.6. Then we let $w=\sum_{j} \phi_{j} w_{j}$. Lemmas 2.4 and 3.6 together with Condition $\mathbf{B}$ and $\mathbf{C}$ then yield

$$
\begin{aligned}
|u-w|_{\hat{H}^{1}(\Omega)}^{2} & \leq C \sum_{j}\left|\phi_{j}\left(u-w_{j}\right)\right|_{\hat{H}^{1}\left(\omega_{j}\right)}^{2} \\
& \leq C \sum\left(\left\|\phi_{j}\right\|_{L^{\infty}}^{2}\left|u-w_{j}\right|_{\hat{H}^{1}\left(\omega_{j}\right)}^{2}+\left\|\nabla \phi_{j}\right\|_{L^{\infty}}^{2}\left\|u-w_{j}\right\|_{L^{2}\left(\omega_{j}\right)}^{2}\right) \\
& \leq C \sum\left(d_{j}^{2 m}\|u\|_{\hat{H}^{m+1}\left(\omega_{j}\right)}^{2}+d_{j}^{2 m}\|u\|_{\hat{H}^{m+1}\left(\omega_{j}\right)}^{2}\right) \leq C h_{\mu}^{2 m}\|u\|_{\hat{H}^{m+1}(\Omega)}^{2},
\end{aligned}
$$

which gives the desired result. $\square$

4. Numerical tests. We close the paper with a numerical application of our method. We chose to test the theoretical predictions on a problem that could be implemented with relative ease, but that presented the approximation challenges discussed in the paper. Our work shows, in particular, that transmission problems give rise to approximation issues similar to those of standard Dirichlet problems. We have therefore tested our results for $\Omega$ the unit disk in two dimensions and the model problem

$$
-\Delta u=4,\left.\quad u\right|_{\partial \Omega}=0,
$$

which has the exact solution $u(x, y)=1-x^{2}-y^{2}$ and allows for more steps in the "refinement." Our partition of unity is obtained by applying the Shepard procedure to radial functions of the form $\tilde{\phi}_{i}(x)=\Phi\left(\left|x-x_{i}\right| / r\right)$. (We suppress the dependence on $\mu$, for the time being, and consider a single GFE space.) The function $\Phi(x)$ is chosen to be $\mathcal{C}^{1}$, and given as follows. It is equal to 1 for $x \leq 1 / 8$, equal to 0 for $x \geq 7 / 8$, and a polynomial of degree 3 for $x \in[1 / 8,7 / 8]$. The resulting partition of unity functions will be denoted $\phi_{i}$. The points $x_{i}$ are chosen on concentric circles of radii $k r, k \in \mathbb{Z}_{+}$. The constant $r$ is chosen to be of the form $r=1 / \mu$, with $\mu>0$ an integer. On each circle, we choose the points to be uniformly distributed at a distance close to $r$, but $\leq r$. Three basis elements in $S_{\mu}: \phi_{i}, x_{1} \phi_{i}$, and $x_{2} \phi_{i}$ will correspond to each point $x_{i}$ that is not on the boundary. On the other hand, exactly one basis element will 


\begin{tabular}{|c|rrrrr|}
\hline$\mu$ & $\mathrm{N}$ & $100 e$ & $N^{2} e$ & $\mu^{2} e$ & $100 e_{a}$ \\
\hline 2 & 35 & 6.6973 & 2.3441 & 0.2679 & 9.8130 \\
\hline 3 & 83 & 3.1637 & 2.6258 & 0.2847 & 4.3541 \\
\hline 4 & 149 & 1.9063 & 2.8403 & 0.3050 & 2.4610 \\
\hline 6 & 336 & 1.0204 & 3.4286 & 0.3673 & 1.1031 \\
\hline 8 & 600 & 0.5365 & 3.2189 & 0.3433 & 0.6228 \\
\hline 12 & 1354 & 0.2601 & 3.5215 & 0.3745 & 0.2779 \\
\hline 16 & 2409 & 0.1353 & 3.2600 & 0.3464 & 0.1567 \\
\hline \multicolumn{5}{c}{ Convergence rates e of GFE solutions the model problem (4.1). }
\end{tabular}

correspond to each $x_{i}$ on the boundary (we choose $[2 \pi / r]+1$ such points, except if $r=1$, in which case we choose 6 such points), using a linear function that vanishes on the tangent to the boundary at $x_{i}$, as in Condition D. We show the results of the numerical test in Table 4.1. In that table, $N$ denotes the number of degrees of freedom, $e$ denotes the $L^{2}$ error, and $e_{a}$ denotes the interpolation error, defined using a Taylor type interpolant as in [5].

The results depend greatly on the numerical quadrature. We have also noticed this issue in an independent implementation of this method in one dimension. See also [2]. We have implemented our method with a less precise quadrature formula, in which case we could run more tests, but the condition $e \leq e_{a}$ was not satisfied any longer.

We conclude that the numerical tests confirm our results, since the scaled errors $N^{2} e$ and $\mu^{2} e$ have the correct behavior (they are uniformly bounded, in fact nearly constant, in our range). This is consistent with the theoretical error estimate $e_{\text {theoretical }} \leq C N^{-2}$. One-dimensional tests show that values $N^{2} e$ are nearly constant in a certain range for the number of the degrees of freedom, after which they start growing rather fast. Replacing the quadrature with a more precise quadrature will delay the moment when $N^{2} e$ starts growing, but does not eliminate this phenomenon ( at least in the one-dimensional tests). A similar phenomenon was noticed in two dimensions (but for a less precise quadrature formula than the one used for the results in Table 4.1), while it does seem to appear for $N^{2} e_{a}$, which is remarkably constant in $N$. The test problem we chose was sufficiently simple that we could implement our method in MATLAB ${ }^{\circledR}$ and run the code on a personal computer (the code is available on the authors' web site.) Even in this case, however, issues of memory management arise due to the growth of degrees of freedom with each "refinement". The approach we have described to the numerical implementation of our method can be applied to transmission problems. Indeed, we have shown that from a theoretical standpoint GFEMs for transmission and Dirichlet problems are handled similarly. At the same time, implementing our method for a transmission problem requires significantly more computing power due to a much larger number of degrees of freedom present in the problem. We are planning more numerical tests, also using other norms for the error, to showcase the method in more general situations.

Acknowledgments. The authors thank J. Melenk for useful references and comments, and the anonymous referees for a careful review of the manuscript and useful suggestions. We also thank S. Brenner for sending us copies of some of her papers. A.L.M. wishes to thank the Institute for Mathematics and its Applications (IMA) for its hospitality during the Program "Complex Fluids and Complex Flows". The IMA 
is partially supported by the National Science Foundation and the University of Minnesota. A.L.M. and Q.Q. were partially supported by NSF Grant DMS-0708902, DMS-1009713. A.L.M. was supported also by NSF Grant DMS-1009714. V.N. was partially supported by the NSF Grants DMS-0713743, OCI-0749202, and DMS1016556. Manuscripts are available from http://www.math.psu.edu/nistor/.

\section{REFERENCES}

[1] I. Babuska, U. Banerjee, and J. Osborn, Generalized finite element methods-main ideas, results and perspective, International Journal of Computational Methods, 1 (2004), pp. 67$1-3$.

[2] I. Babuska, U. Banerjee, J. Osborn, And Q. Li, Quadrature for meshless methods, International Journal for Numerical Methods in Engineering, 76 (2008).

[3] I. BABuška, U. BAnERJEe, And J. E. Osborn, Survey of meshless and generalized finite element methods: A unified approach, Acta Numerica, 12 (2003), pp. 1-125.

[4] I. Babuska And J. Melenk, The partition of unity method, International Journal for Numerical Methods in Engineering, 40 (1997), pp. 727-758.

[5] I. BABUŠKA AND V. NiSTOR, Interior numerical approximation of boundary value problems with a distributional data, Numer. Methods Partial Differential Equations, 22 (2006), pp. 79113.

[6] I. Babuska, V. Nistor, and N. Tarfulea, Approximate and low regularity Dirichlet Boundary Conditions in the Generalized Finite Element Method (PREPRINT), Math. Models Methods Appl. Sci, 17 (2007), pp. 2115-2142.

[7] I. BabušKa, V. Nistor, And N. TARfulea, Generalized finite element method for second-order elliptic operators with dirichlet boundary conditions, J. Comput. Appl. Math., 218 (2008), pp. 175-183.

[8] C. Bacuta And J. Sun, Partition of unity finite element method implementation for Poisson equation, Advances in Applied and Computational Mathematics, Nova Publishers, New York, (2006), pp. 35-46.

[9] J. BARRett AND C. Elliott, Finite element approximation of the Dirichlet problem using the boundary penalty method, Numer. Math., 49 (1986), pp. 343-366.

[10] - Fitted and unfitted finite-element methods for elliptic equations with smooth interfaces, IMA J. Numer. Anal., 7 (1987), pp. 283-300.

[11] A. Berger, R. Scott, and G. Strang, Approximate boundary conditions in the finite element method, in Symposia Mathematica, vol. 10, 1972, pp. 295-313.

[12] S. Brenner, Poincaré-Friedrichs inequalities for piecewise $H^{1}$ functions, SIAM J. Numer. Anal., 41 (2003), pp. 306-324 (electronic).

[13] S. Brenner And L. Sung, Multigrid methods for the computation of singular solutions and stress intensity factors. III. Interface singularities, Comput. Methods Appl. Mech. Engrg., 192 (2003), pp. 4687-4702.

[14] S. Brenner, K. Wang, And J. Zhao, Poincaré-Friedrichs inequalities for piecewise $H^{2}$ functions, Numer. Funct. Anal. Optim., 25 (2004), pp. 463-478.

[15] S. C. Brenner And L. R. ScotT, The mathematical theory of finite element methods, vol. 15 of Texts in Applied Mathematics, Springer-Verlag, New York, second ed., 2002.

[16] E. Chung, Q. Du, And J. Zou, Convergence analysis of a finite volume method for Maxwell's equations in nonhomogeneous media, SIAM Journal on Numerical Analysis, 41 (2004), pp. $37-63$.

[17] P. G. Ciarlet, The finite element method for elliptic problems, vol. 40 of Classics in Applied Mathematics, Society for Industrial and Applied Mathematics (SIAM), Philadelphia, PA, 2002. Reprint of the 1978 original [North-Holland, Amsterdam; MR0520174 (58 \#25001)].

[18] J. Dolbow, N. Moes, and T. Belytschko, An extended finite element method for modeling crack growth with frictional contact, Computer Methods in Applied Mechanics and Engineering, 190 (2001), pp. 6825-6846.

[19] C. Dunkte, O. Hamzeh, T. Liszka, and W. Tworzydlo, A generalized finite element method for the simulation of three-dimensional dynamic crack propagation, Computer Methods in Applied Mechanics and Engineering, 190 (2001), pp. 2227-2262.

[20] M. Griebel And M. Schweitzer, A particle-partition of unity method for the solution of elliptic, parabolic, and hyperbolic pdes, SIAM Journal on Scientific Computing, 22 (2000), pp. 853-890.

[21] M. Griebel And M. Schweitzer, A particle-partition of unity method, Part V: Boundary 
conditions, Geometric Analysis and Nonlinear Partial Differential Equations, 41 (2002), pp. $115-137$.

[22] A. Hansbo and P. Hansbo, An unfitted finite element method, based on Nitsche's method, for elliptic interface problems, Computer methods in applied mechanics and engineering, 191 (2002), pp. 5537-5552.

[23] R. KellogG, Singularities in interface problems, Numerical solution of partial differential equations II, (1971), pp. 351-400.

[24] R. LEVEQUE AND Z. LI, The immersed interface method for elliptic equations with discontinuous coefficients and singular sources, SIAM Journal on Numerical Analysis, (1994), pp. 10191044.

[25] H. LI, A note on the conditioning of a class of generalized finite element methods. 2010.

[26] H. Li, A. Mazzucato, And V. Nistor, Analysis of the finite element method for transmission/mixed boundary value problems on general polygonal domains, Electron. Trans. Numer. Anal., 37 (2010), pp. 41-69.

[27] J. Li, J. M. Melenk, B. Wohlmuth, And J. Zou, Optimal a priori estimates for higher order finite elements for elliptic interface problems, Appl. Numer. Math., 60 (2010), pp. 19-37.

[28] Z. Li AND S. LubKin, Numerical analysis of interfacial two-dimensional Stokes flow with discontinuous viscosity and variable surface tension, International Journal for Numerical Methods in Fluids, 37 (2001).

[29] S. NicAise AND A. SANDig, Transmission problems for the Laplace and elasticity operators: Regularity and boundary integral formulation, Mathematical Models and Methods in Applied Sciences, 9 (1999), pp. 855-898.

[30] J. Nitsche, On Dirichlet problems using subspaces with nearly zero boundary conditions, The Mathematical Foundations of the Finite Element Method with Applications to Partial Differential Equations, (1972), pp. 603-627.

[31] C. Peskin, Numerical analysis of blood flow in the heart, J. Comput. Phys, 25 (1977), pp. 220252.

[32] Y. Roitberg and Z. Sheftel, General boundary-value problems for elliptic equations with discontinuous coefficients, in Dokl. Akad. Nauk SSSR, vol. 148, 1963, pp. 1034-1037.

[33] R. SсотT, Interpolated boundary conditions in the finite element method, SIAM Journal on Numerical Analysis, 12 (1975), pp. 404-427.

[34] A. Simone, C. Duarte, and E. Van Der Giessen, A generalized finite element method for polycrystals with discontinuous grain boundaries, Int. J. Numer. Meth. Engng, 67 (2006), pp. 1122-1145.

[35] R. K. Sinha and B. DekA, On the convergence of finite element method for second order elliptic interface problems, Numer. Funct. Anal. Optim., 27 (2006), pp. 99-115.

[36] E. M. Stein, Singular integrals and differentiability properties of functions, Princeton Mathematical Series, No. 30, Princeton University Press, Princeton, N.J., 1970.

[37] T. Strouboulis, I. BABuŠKA, AND K. COPPS, The design and analysis of the generalized finite element method, Computer methods in applied mechanics and engineering, 181 (2000), pp. 43-69.

[38] N. Sukumar, D. Chopp, And B. Moran, Extended finite element method and fast marching method for three-dimensional fatigue crack propagation, Engineering Fracture Mechanics, 70 (2003), pp. 29-48.

[39] M. E. TAYlor, Partial differential equations I. Basic theory, vol. 115 of Applied Mathematical Sciences, Springer, New York, second ed., 2011.

[40] A. V.Nistor, Well-posedness and regularity for the elasticity equation with mixed boundary conditions on polyhedral domains and domains with cracks, Archive for Rational Mechanics and Analysis, online (2008).

[41] J. Xu, Error estimates of the finite element method for the 2nd order elliptic equation with discontinuous coefficient, J. Xiangtan Univ, (1982).

[42] J. XU AND Y. ZHU, Uniform convergent multigrid methods for elliptic problems with strongly discontinuous coefficients, Mathematical Models and Methods in Applied Science, 18 (2008), pp. $77-105$.

[43] Y. ZHu, Domain decomposition preconditioners for elliptic equations with jump coefficients, Numerical Linear Algebra with Applications, 15 (2008). 